\title{
Home-Based Tele-Exercise in Musculoskeletal Conditions and Chronic Disease: A Literature Review
}

OPEN ACCESS

Edited by:

Feng Yang,

Georgia State University,

United States

Reviewed by:

Xuan Liu,

Kessler Foundation, United States

Diego Ferreira,

Lebanon Valley College, United States

*Correspondence:

Alice S. Ryan

aryan@som.umaryland.edu

Specialty section:

This article was submitted to Rehabilitation for Musculoskeletal

Conditions,

a section of the journa

Frontiers in Rehabilitation Sciences

Received: 08 November 2021

Accepted: 28 January 2022

Published: 24 February 2022

Citation:

Amorese AJ and Ryan AS (2022)

Home-Based Tele-Exercise in

Musculoskeletal Conditions and

Chronic Disease: A Literature Review.

Front. Rehabilit. Sci. 3:811465.

doi: $10.3389 /$ fresc. 2022.811465

\begin{abstract}
Adam J. Amorese ${ }^{1}$ and Alice S. Ryan ${ }^{1,2 *}$
${ }^{1}$ Baltimore Veterans Affairs (VA) Medical Center, Geriatric Research, Education and Clinical Center (GRECC), VA Maryland Health Care System, Baltimore, MD, United States, ${ }^{2}$ VA Research Service, Baltimore GRECC, Department of Medicine,

Division of Geriatrics and Palliative Medicine, University of Maryland School of Medicine, Baltimore, MD, United States
\end{abstract}

Exercise training is an essential component in the treatment or rehabilitation of various diseases and conditions. However, barriers to exercise such as the burdens of travel or time may hinder individuals' ability to participate in such training programs. Advancements in technology have allowed for remote, home-based exercise training to be utilized as a supplement or replacement to conventional exercise training programs. Individuals in these home-based exercise programs are able to do so under varying levels of supervision from trained professionals, with some programs having direct supervision, and others having little to no supervision at all. The purpose of this review is to examine the use of home-based, tele-exercise training programs for the treatment of different disease states and conditions, and how these programs compare to conventional clinic-based exercise training programs.

Keywords: tele-exercise, home-based exercise, remote exercise, telerehabilitation, rehabilitation, exercise training

\section{INTRODUCTION}

Although, exercise is a vital component in the treatment or prevention of numerous diseases and conditions, barriers to exercise exist in many populations (1-5). These barriers include inaccessibility to facilities, time constraints, and the cost of programs (6). One option to overcome these barriers is to implement home-based tele-exercise training to alleviate these burdens that may hinder participation in exercise programs. With advancements in technology such as high-speed internet, video-conferencing software, and smartphones, exercise training can be performed remotely under the supervision of experienced, trained professionals. Additionally, training programs may utilize methods which involve exercise with limited supervision by trained professionals, or even no supervision at all (Figure 1). These remote, tele-exercise programs may be useful in a variety of diseases or conditions, including musculoskeletal issues, diseases of the cardiorespiratory system, or neurological conditions. The following review will discuss studies employing tele-exercise training programs for the treatment and rehabilitation of these conditions and diseases.

\section{LITERATURE SEARCH}

A literature search was performed from December 2020 to March 2021 on PubMed and Google Scholar with key words including but not limited to "tele-exercise," "telerehabilitation," and "remote exercise." Reference lists of relevant articles were also searched. Studies included were published 
between 2008 and 2021 and involved home-based exercise under direct supervision during exercise, indirect supervision with some sort of contact between participants and research team members during the exercise intervention, or no supervision at all during exercise. In total, 59 studies were reviewed, with 40 of these studies being randomized control trials (RCT). Studies were organized into the following three groups: Musculoskeletal (conditions or diseases pertaining to the lower and upper extremities) (Table 1), Cardiorespiratory (conditions or diseases pertaining to cardiovascular or respiratory health) (Table 2), or Neurological (conditions or diseases pertaining to the nervous system) (Table 3 ).

\section{MUSCULOSKELETAL}

With impairments of the joints, musculoskeletal pain and hindered mobility can occur, especially in older individuals (7, 8). These impairments can include chronic ailments, such as osteoarthritis (OA), or acute injury. Following injury and repair of the joints of the lower and upper extremities, exercise is considered a critical part of the rehabilitation process (9-12). Ultimately, the goal of these exercises is to improve strength, mobility, and balance, as well as manage pain $(13,14)$.

Home-based exercises under varying levels of supervision have been used in studies of rehabilitation for impairments to the lower extremities, specifically total knee arthroplasty (TKA). Three RCTs have utilized functional or strengthening exercises under direct supervision, comparing outcomes in telerehabilitation vs. outpatient or home-visit rehabilitation (15-18). No differences in improvements were observed between groups for outcomes including the Western Ontario and McMaster Universities Osteoarthritis Index (WOMAC), range of motion, muscle strength, and walking ability. One of the studies also performed a cost analysis of the intervention, which showed a lower mean cost per session in the telerehabilitation group compared to the control group, mainly due to the cost of travel for those further away from the health care center (18). One RCT with limited supervision of exercise has been performed with individuals who had TKA in which they had unrestricted use (frequency and duration) of a novel virtual physical therapy system (19). Exercises were preloaded into the system and progress was monitored remotely. Following the intervention, no significant differences were observed between those using the system and those receiving usual care in knee extension and flexion, pain, gait speed, or physical function. Heath care costs were significantly lower for those receiving virtual therapy compared to those receiving clinic-based therapy in the usual care group due to significantly fewer home health and outpatient physical therapy visits and inpatient rehospitalizations (19). One other study in individuals with TKA was done using telerehabilitation without supervision, using prerecorded videos of exercises provided to participants (20). No significant differences were observed between the telerehabilitation group and those receiving conventional rehabilitation for any clinical outcomes measured including scales of pain and physical function. Additionally, satisfaction with care was similar between groups, while utilization of hospital-based resources was $60 \%$ lower in the telerehabilitation group (20). With all of these RCTs of individuals who had TKA, a telerehabilitation group was compared to conventional outpatient rehabilitation. Independent of the level of supervision, intervention duration, or type of exercise used, results showed similar improvements in outcomes between telerehabilitation and outpatient rehabilitation groups. This suggests that using telerehabilitation exercise that follow conventional, outpatient rehabilitation methodology is a comparable option for rehabilitation treatment in individuals with TKA.

Telerehabilitation studies have also been conducted in individuals with other ailments of the lower limb, including $\mathrm{OA}$, knee or hip replacement, and acute injury. For individuals with $\mathrm{OA}$, exercises used in telerehabilitation studies include

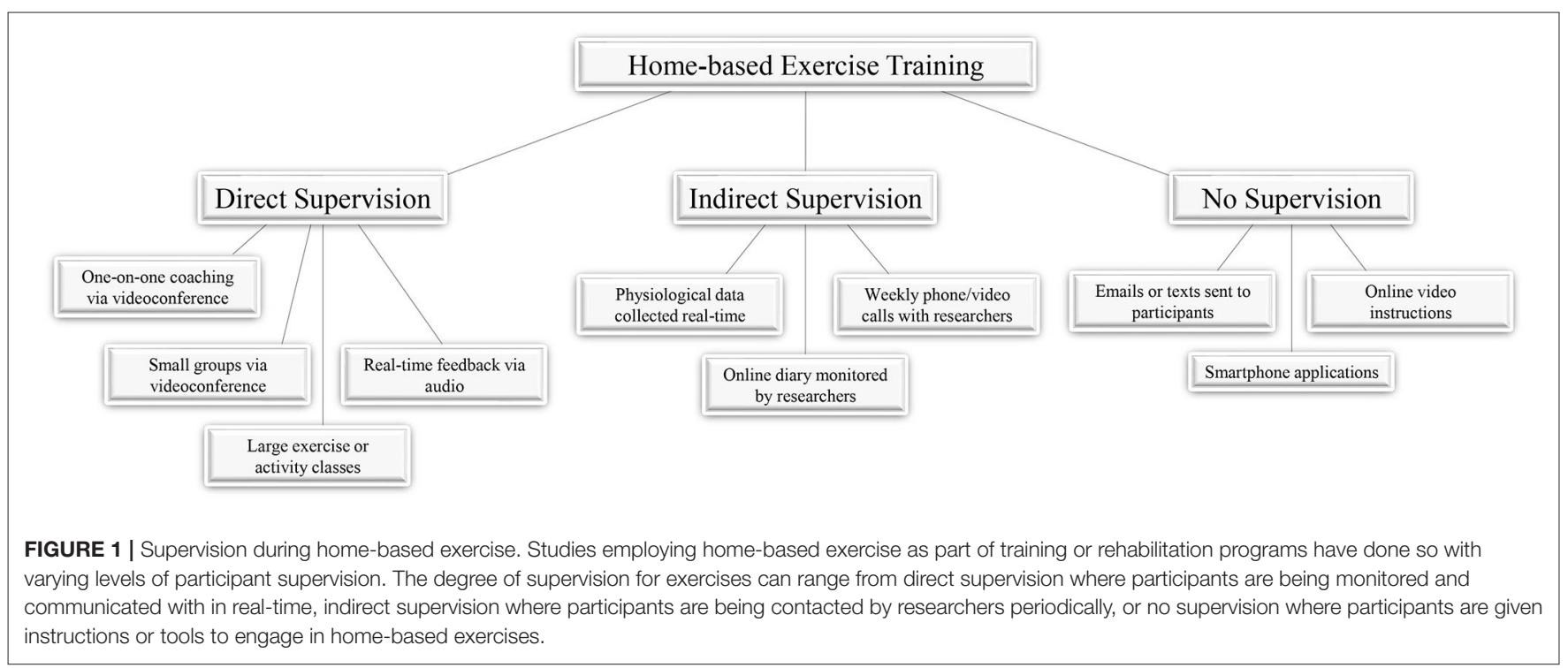


TABLE 1 | Characteristics and outcomes of home-based tele-exercise studies - musculoskeletal system.

\begin{tabular}{|c|c|c|c|c|c|c|c|c|}
\hline Research study & $\begin{array}{l}\text { RCT/ } \\
\text { population/ } \\
\text { sample size }\end{array}$ & $\begin{array}{l}\text { Mode of delivery/ } \\
\text { exercise }\end{array}$ & $\begin{array}{l}\text { Intervention } \\
\text { duration }\end{array}$ & Comparator groups & $\begin{array}{l}\text { Training } \\
\text { frequency }\end{array}$ & $\begin{array}{l}\text { Training } \\
\text { duration }\end{array}$ & Follow-up & Results \\
\hline Russell et al. (15) & $\mathrm{Y} / \mathrm{TKA} / n=65$ & $\begin{array}{l}\text { Direct supervision, webcam; } \\
\text { functional exercises }\end{array}$ & 6 weeks & $\begin{array}{l}\text { Telerehabilitation vs. } \\
\text { outpatient rehabilitation }\end{array}$ & $1 \times /$ week & $45 \mathrm{~min}$ & No follow-up & $\begin{array}{l}\text { No difference in improvements in range of } \\
\text { motion, muscle strength, pain, timed } \\
\text { up-and-go test, quality of life, and clinical } \\
\text { gait scores }\end{array}$ \\
\hline $\begin{array}{l}\text { Tousignant et al. } \\
\text { (16) }\end{array}$ & $\mathrm{Y} / \mathrm{TKA} / n=48$ & $\begin{array}{l}\text { Direct supervision, webcam; } \\
\text { functional exercises }\end{array}$ & 8 weeks & $\begin{array}{l}\text { Telerehabilitation vs. } \\
\text { outpatient/home visit } \\
\text { rehabilitation }\end{array}$ & $2 \times /$ week & $1 \mathrm{~h}$ & No follow-up & $\begin{array}{l}\text { No difference in improvements in } \\
\text { WOMAC, range of motion, balance, and } \\
\text { lower body strength }\end{array}$ \\
\hline $\begin{array}{l}\text { Moffet et al. (17), } \\
\text { Tousignant et al. } \\
\text { (18) }\end{array}$ & $\mathrm{Y} / \mathrm{TKA} / n=205$ & $\begin{array}{l}\text { Direct supervision, webcam; } \\
\text { mobility, strengthening, } \\
\text { balance, and functional } \\
\text { exercises }\end{array}$ & 8 weeks & $\begin{array}{l}\text { Telerehabilitation vs. home } \\
\text { visit rehabilitation }\end{array}$ & $2 \times /$ week & $45-60 \mathrm{~min}$ & No follow-up & $\begin{array}{l}\text { No difference in improvements in } \\
\text { WOMAC, range of motion, and 6MWT; } \\
\text { lower costs compared to control group }\end{array}$ \\
\hline $\begin{array}{l}\text { Prvu Bettger et al. } \\
\text { (19) }\end{array}$ & $\mathrm{Y} / \mathrm{TKA} / n=306$ & $\begin{array}{l}\text { Indirect supervision, weekly } \\
\text { videoconference; physical } \\
\text { therapy exercises }\end{array}$ & 12 weeks & $\begin{array}{l}\text { Telerehabilitation vs. } \\
\text { outpatient/home visit } \\
\text { rehabilitation }\end{array}$ & Unrestricted & Unrestricted & No follow-up & $\begin{array}{l}\text { No significant differences between } \\
\text { telerehabilitation and usual care in } \\
\text { functional outcomes; lower health care } \\
\text { costs with telerehabilitation }\end{array}$ \\
\hline $\begin{array}{l}\text { Bini and Mahajan } \\
\text { (20) }\end{array}$ & $\mathrm{Y} / \mathrm{TKA} / n=29$ & $\begin{array}{l}\text { No supervision, } \\
\text { pre-recorded videos; } \\
\text { physical therapy exercises }\end{array}$ & 12 weeks & $\begin{array}{l}\text { Telerehabilitation vs. } \\
\text { outpatient rehabilitation }\end{array}$ & $\begin{array}{l}\text { No set number of } \\
\text { sessions }\end{array}$ & Not reported & No follow-up & $\begin{array}{l}\text { No differences in improvements in clinical } \\
\text { outcomes between groups; similar } \\
\text { satisfaction, but lower hospital-based } \\
\text { resource utilization in telerehabilitation } \\
\text { group }\end{array}$ \\
\hline Hinman et al. (21) & $\mathrm{N} / \mathrm{OA} / n=12$ & $\begin{array}{l}\text { Direct supervision, webcam; } \\
\text { strengthening exercises }\end{array}$ & 3 months & $\begin{array}{l}\text { Telerehabilitation only, no } \\
\text { control group }\end{array}$ & $\begin{array}{l}7 \text { total webcam } \\
\text { sessions, } \\
3 \times / \text { week training } \\
\text { sessions }\end{array}$ & Not reported & $\begin{array}{l}3-6 \text { months } \\
\text { following } \\
\text { rehabilitation }\end{array}$ & $\begin{array}{l}\text { Self-reported reductions in knee pain and } \\
\text { high satisfaction }\end{array}$ \\
\hline Azma et al. (22) & $\begin{array}{l}\text { Y/knee OA/n = } \\
54\end{array}$ & $\begin{array}{l}\text { Indirect supervision, } \\
\text { telephone; strengthening, } \\
\text { endurance, flexibility, active } \\
\text { range of motion exercises }\end{array}$ & 6 weeks & $\begin{array}{l}\text { Telerehabilitation vs. } \\
\text { outpatient rehabilitation }\end{array}$ & $3 \times /$ week & Not reported & $\begin{array}{l}1 \text { and } 6 \\
\text { months }\end{array}$ & $\begin{array}{l}\text { No difference in improvements in KOOS } \\
\text { and WOMAC scores between groups at all } \\
\text { time points }\end{array}$ \\
\hline \multirow[t]{3}{*}{ Chang et al. (23) } & $\begin{array}{l}\mathrm{N} / \mathrm{hip} \\
\text { replacement } / n= \\
31\end{array}$ & $\begin{array}{l}\text { Indirect supervision, } \\
\text { telephone; range of motion, } \\
\text { resistance training, walking }\end{array}$ & 12 weeks & $\begin{array}{l}\text { Intervention group only (pilot } \\
\text { study) }\end{array}$ & $\begin{array}{l}\text { Range of motion: } \\
3 \times / \text { day }\end{array}$ & $\begin{array}{l}\text { Range of } \\
\text { motion: } 10 \mathrm{~min}\end{array}$ & No follow-up & $\begin{array}{l}\text { Significant improvements in TUG, 6MWT, } \\
\text { and WOMAC; high satisfaction and } \\
\text { feasibility }\end{array}$ \\
\hline & & & & & $\begin{array}{l}\text { Resistance: } 1 \times \\
\text { every other day }\end{array}$ & $\begin{array}{l}\text { Resistance: } \\
10 \mathrm{~min}\end{array}$ & & \\
\hline & & & & & Walking: $1 \times /$ day & $\begin{array}{l}\text { Walking: } \\
\text { 10-30 min }\end{array}$ & & \\
\hline $\begin{array}{l}\text { Eichler et al. } \\
(24,25)\end{array}$ & $\begin{array}{l}\text { Y/knee or hip } \\
\text { replacement } / n= \\
111\end{array}$ & $\begin{array}{l}\text { Indirect supervision, text, } \\
\text { telephone, and } \\
\text { videoconference; strength } \\
\text { and postural control } \\
\text { exercises following inpatient } \\
\text { rehabilitation }\end{array}$ & 3 months & $\begin{array}{l}\text { Telerehabilitation and usual } \\
\text { care vs. usual care alone }\end{array}$ & $3 \times /$ week & Not reported & No follow-up & $\begin{array}{l}\text { No difference in improvements between } \\
\text { groups in functional tests such as timed } \\
\text { up-and-go, } 6 \mathrm{MWT} \text {, and stair ascent test }\end{array}$ \\
\hline
\end{tabular}


TABLE 1 | Continued

\begin{tabular}{|c|c|c|c|c|c|c|c|c|}
\hline Research study & $\begin{array}{l}\text { RCT/ } \\
\text { population/ } \\
\text { sample size }\end{array}$ & $\begin{array}{l}\text { Mode of delivery/ } \\
\text { exercise }\end{array}$ & $\begin{array}{l}\text { Intervention } \\
\text { duration }\end{array}$ & Comparator groups & $\begin{array}{l}\text { Training } \\
\text { frequency }\end{array}$ & $\begin{array}{l}\text { Training } \\
\text { duration }\end{array}$ & Follow-up & Results \\
\hline $\begin{array}{l}\text { Tsvyakh and } \\
\text { Hospodarskyy (26) }\end{array}$ & $\begin{array}{l}\text { N/Lower } \\
\text { extremity injury/n } \\
=74\end{array}$ & $\begin{array}{l}\text { Indirect supervision, } \\
\text { smartphone sensors; } \\
\text { passive and active } \\
\text { flexion-extension, } \\
\text { dosed-load }\end{array}$ & 3 months & $\begin{array}{l}\text { Telerehabilitation vs. } \\
\text { outpatient rehabilitation }\end{array}$ & $\begin{array}{l}\text { Individualized to } \\
\text { patient }\end{array}$ & $\begin{array}{l}\text { Individualized to } \\
\text { patient }\end{array}$ & No follow-up & $\begin{array}{l}\text { Higher patient satisfaction in } \\
\text { telerehabilitation group compared to } \\
\text { traditional rehab }\end{array}$ \\
\hline $\begin{array}{l}\text { Pastora-Bernal } \\
\text { et al. }(27,28)\end{array}$ & $\mathrm{Y} / \mathrm{ASD} / n=18$ & $\begin{array}{l}\text { No supervision, web-based } \\
\text { videos and images }\end{array}$ & 12 weeks & $\begin{array}{l}\text { Telerehabilitation vs. } \\
\text { outpatient rehabilitation }\end{array}$ & $5 \times /$ week & Not reported & No follow-up & $\begin{array}{l}\text { No difference in improvements in shoulder } \\
\text { function between groups }\end{array}$ \\
\hline Choi et al. (29) & $\begin{array}{l}\text { Y/frozen } \\
\text { shoulder/n = } 84\end{array}$ & $\begin{array}{l}\text { No supervision, } \\
\text { smartphone-assisted; } \\
\text { flexion, rotation, adduction, } \\
\text { stretching }\end{array}$ & 12 weeks & $\begin{array}{l}\text { Smartphone-assisted } \\
\text { exercise vs. conventional } \\
\text { exercise }\end{array}$ & $2-3 \times /$ day & $\begin{array}{l}\text { Each exercise } \\
10 \times\end{array}$ & No follow-up & $\begin{array}{l}\text { No difference in improvements in shoulder } \\
\text { pain or range of motion between groups }\end{array}$ \\
\hline Santello et al. (30) & $\begin{array}{l}\text { Y/shoulder } \\
\text { pain } / n=60\end{array}$ & $\begin{array}{l}\text { No supervision, web-based } \\
\text { videos, instruction booklet; } \\
\text { stretching, joint mobility, } \\
\text { strengthening exercises }\end{array}$ & 2 months & $\begin{array}{l}\text { Home-based rehabilitation } \\
\text { vs. control group receiving } \\
\text { minimal education only }\end{array}$ & $3 \times /$ week & $\begin{array}{l}3-5 \text { sets of } \\
\text { exercises, } 5-10 \\
\text { repetitions }\end{array}$ & No follow-up & $\begin{array}{l}\text { Significant improvements in pain and } \\
\text { disability compared to no rehabilitation }\end{array}$ \\
\hline \multirow[t]{2}{*}{ Malliaras et al. (31) } & $\begin{array}{l}\text { Y/rotator cuff } \\
\text { pain } / n=36\end{array}$ & $\begin{array}{l}\text { Direct supervision, webcam; } \\
\text { range of motion exercises }\end{array}$ & 12 weeks & $\begin{array}{l}\text { Advice only vs. } \\
\text { recommended care vs. } \\
\text { recommended care with } \\
\text { telerehabilitation }\end{array}$ & $\begin{array}{l}1 \times / \text { week } \\
\text { (telerehabilitation } \\
\text { sessions) }\end{array}$ & 30-60 min & No follow-up & $\begin{array}{l}\text { High retention and acceptable adherence } \\
\text { with telerehabilitation; general } \\
\text { improvement in indices of pain and } \\
\text { function }\end{array}$ \\
\hline & & & & & $\begin{array}{l}3 \times / \text { week } \\
\text { (recommended } \\
\text { care) }\end{array}$ & & & \\
\hline $\begin{array}{l}\text { Eriksson et al. } \\
(32,33)\end{array}$ & $\begin{array}{l}\mathrm{N} / \text { shoulder OA/n } \\
=25\end{array}$ & $\begin{array}{l}\text { Direct supervision, webcam; } \\
\text { physical therapy exercises }\end{array}$ & 8 weeks & $\begin{array}{l}\text { Telerehabilitation vs. } \\
\text { outpatient rehabilitation }\end{array}$ & $1-3 \times /$ week & $30-60 \mathrm{~min}$ & No follow-up & $\begin{array}{l}\text { Greater improvements in pain and } \\
\text { shoulder function in telerehabilitation group }\end{array}$ \\
\hline $\begin{array}{l}\text { Tousignant et al. } \\
\text { (34) }\end{array}$ & $\begin{array}{l}\mathrm{N} / \text { fracture to } \\
\text { proximal } \\
\text { humerus } / n=17\end{array}$ & $\begin{array}{l}\text { Direct supervision, webcam; } \\
\text { stretching, pain control, } \\
\text { range of motion, } \\
\text { strengthening exercises }\end{array}$ & 8 weeks & $\begin{array}{l}\text { Telerehabilitation only, no } \\
\text { control group }\end{array}$ & $1-2 \times /$ week & $30-45 \mathrm{~min}$ & No follow-up & $\begin{array}{l}\text { Decrease in pain and improvements in } \\
\text { range of motion and function; high } \\
\text { satisfaction }\end{array}$ \\
\hline Steiner et al. (35) & $\begin{array}{l}\mathrm{N} / \text { chronic } \\
\text { shoulder } \\
\text { disease } / n=12\end{array}$ & $\begin{array}{l}\text { Indirect supervision, } \\
\text { Microsoft Kinect, telephone; } \\
\text { physiotherapeutic exercises }\end{array}$ & 3 months & $\begin{array}{l}\text { Telerehabilitation group only, } \\
\text { no control group }\end{array}$ & $5 \times /$ week, $2 \times /$ day & $10-15 \min$ & No follow-up & $\begin{array}{l}\text { High useability and satisfaction; improved } \\
\text { range of motion }\end{array}$ \\
\hline
\end{tabular}


TABLE 2 | Characteristics and outcomes of home-based tele-exercise studies-cardiorespiratory.

\begin{tabular}{|c|c|c|c|c|c|c|c|c|}
\hline Research study & $\begin{array}{l}\text { RCT/ } \\
\text { Population/ } \\
\text { Sample Size }\end{array}$ & $\begin{array}{l}\text { Mode of delivery/ } \\
\text { exercise }\end{array}$ & $\begin{array}{l}\text { Intervention } \\
\text { duration }\end{array}$ & Comparator groups & $\begin{array}{l}\text { Training } \\
\text { frequency }\end{array}$ & $\begin{array}{l}\text { Training } \\
\text { duration }\end{array}$ & Follow-up & Results \\
\hline $\begin{array}{l}\text { Maddison et al. } \\
(39,40) \text {, Rawstorn } \\
\text { et al. (41) }\end{array}$ & $\begin{array}{l}\text { Y/coronary heart } \\
\text { disease } / n=162\end{array}$ & $\begin{array}{l}\text { Direct supervision, audio } \\
\text { communication, } \\
\text { smartphone and web apps; } \\
\text { walking at } 40-65 \% \text { of heart } \\
\text { rate reserve }\end{array}$ & 12 weeks & $\begin{array}{l}\text { Telerehabilitation vs. } \\
\text { outpatient rehabilitation }\end{array}$ & $3 \times /$ week & $30-60 \mathrm{~min}$ & 12 weeks & $\begin{array}{l}\text { Similar increases in } \mathrm{VO}_{2} \text { max between } \\
\text { groups, telerehabilitation group less } \\
\text { sedentary at } 12 \text {-week follow-up; high } \\
\text { acceptability and useability of program }\end{array}$ \\
\hline Kraal et al. $(42,43)$ & $\begin{array}{l}\text { Y/coronary heart } \\
\text { disease } / n=90\end{array}$ & $\begin{array}{l}\text { Indirect supervision, } \\
\text { telephone; aerobic exercise }\end{array}$ & 12 weeks & $\begin{array}{l}\text { Home-based rehabilitation } \\
\text { vs. center-based } \\
\text { rehabilitation }\end{array}$ & $2 \times /$ week & $45-60 \mathrm{~min}$ & 1 year & $\begin{array}{l}\text { Similar significant improvements in peak } \\
\mathrm{VO}_{2} \text { sustained at 1-year follow-up; lower } \\
\text { costs in home-based group }\end{array}$ \\
\hline $\begin{array}{l}\text { Hwang et al. } \\
(44,45)\end{array}$ & $\mathrm{Y} / \mathrm{CHF} / n=53$ & $\begin{array}{l}\text { Direct monitoring, webcam; } \\
\text { aerobic and strength } \\
\text { exercises }\end{array}$ & 12 weeks & $\begin{array}{l}\text { Telerehabilitation vs. } \\
\text { outpatient rehabilitation }\end{array}$ & $2 \times /$ week & $60 \mathrm{~min}$ & 12 weeks & $\begin{array}{l}\text { No difference in non-significant 6MWD } \\
\text { improvement between groups; lower } \\
\text { costs for home-based group }\end{array}$ \\
\hline $\begin{array}{l}\text { Bravo-Escobar } \\
\text { et al. ( } 46)\end{array}$ & $\begin{array}{l}\text { Y/coronary } \\
\text { artery disease } / n \\
=28\end{array}$ & $\begin{array}{l}\text { No supervision, remote, } \\
\text { ECG device; treadmill and } \\
\text { stationary bike, resistance } \\
\text { training }\end{array}$ & 2 months & $\begin{array}{l}\text { Home-based mixed } \\
\text { surveillance rehabilitation vs. } \\
\text { outpatient rehabilitation }\end{array}$ & $3 \times /$ week & $1 \mathrm{~h}$ & No follow-up & $\begin{array}{l}\text { Significant improvements in exercise time } \\
\text { and capacity in both groups }\end{array}$ \\
\hline Fang et al. (47) & $\begin{array}{l}\text { Y/percutaneous } \\
\text { coronary } \\
\text { intervention } / n= \\
67\end{array}$ & $\begin{array}{l}\text { Indirect supervision, } \\
\text { telephone; walking/jogging }\end{array}$ & 6 weeks & $\begin{array}{l}\text { Telerehabilitation vs. usual } \\
\text { care }\end{array}$ & $3 \times /$ week & Not reported & No follow-up & $\begin{array}{l}\text { Significantly greater improvements in } \\
6 \mathrm{MWT} \text { and quality of life with } \\
\text { telerehabilitation }\end{array}$ \\
\hline $\begin{array}{l}\text { Maddison et al. } \\
(48,50,51) \text {, } \\
\text { Pfaeffli Dale et al. } \\
\text { (49) }\end{array}$ & $\begin{array}{l}\text { Y/coronary heart } \\
\text { disease/ } n=171\end{array}$ & $\begin{array}{l}\text { No supervision, text } \\
\text { messages; moderate to } \\
\text { vigorous aerobic exercise }\end{array}$ & 24 weeks & $\begin{array}{l}\text { Mobile health rehabilitation } \\
\text { vs. usual care }\end{array}$ & $5 \times /$ week & $30 \mathrm{~min}$ & No follow-up & $\begin{array}{l}\text { No changes to peak } \mathrm{VO}_{2} \text { in either group; } \\
\text { significant increase in leisure time activity } \\
\text { and walking in mobile health group }\end{array}$ \\
\hline $\begin{array}{l}\text { Frederix et al. } \\
(52,53)\end{array}$ & $\begin{array}{l}\text { Y/coronary } \\
\text { artery disease, } \\
\mathrm{CHF} / n=126\end{array}$ & $\begin{array}{l}\text { No supervision, email and } \\
\text { text messages; aerobic } \\
\text { exercise }\end{array}$ & 6 months & $\begin{array}{l}\text { Center-based cardiac } \\
\text { rehabilitation plus } \\
\text { telerehabilitation vs. } \\
\text { center-based cardiac } \\
\text { rehabilitation alone }\end{array}$ & $3 \times /$ week & At least $30 \mathrm{~min}$ & 2 years & $\begin{array}{l}\text { Significant improvement in peak } \mathrm{VO}_{2} \text { in } \\
\text { telerehabilitation group, but lost at 2-year } \\
\text { follow-up; progressive decline in peak } \mathrm{VO}_{2} \\
\text { in center-based group after } 6 \text { months and } \\
\text { 2-year follow-up }\end{array}$ \\
\hline $\begin{array}{l}\text { Holland et al. } \\
(60,61)\end{array}$ & $\begin{array}{l}\mathrm{Y} / \mathrm{COPD} / \mathrm{n}= \\
166\end{array}$ & $\begin{array}{l}\text { Indirect supervision, } \\
\text { telephone; walking, cycling, } \\
\text { resistance training }\end{array}$ & 8 weeks & $\begin{array}{l}\text { Home-based rehabilitation } \\
\text { vs. center-based } \\
\text { rehabilitation }\end{array}$ & $2 \times /$ week & At least $30 \mathrm{~min}$ & 12 months & $\begin{array}{l}\text { Similar improvements in } 6 \mathrm{MWD} \text { and } \\
\text { quality of life outcomes, lost at 12-month } \\
\text { follow-up }\end{array}$ \\
\hline \multirow[t]{2}{*}{$\begin{array}{l}\text { Hansen et al. } \\
(62,63) \text {, } \\
\text { Godtfredsen et al. } \\
(64)\end{array}$} & $\begin{array}{l}\mathrm{Y} / \mathrm{COPD} / \mathrm{n}= \\
134\end{array}$ & $\begin{array}{l}\text { Direct supervision, webcam; } \\
\text { endurance and resistance } \\
\text { training }\end{array}$ & 10 weeks & $\begin{array}{l}\text { Telerehabilitation vs. } \\
\text { hospital-based rehabilitation }\end{array}$ & $\begin{array}{l}\text { Telerehabilitation: } \\
3 \times / \text { week }\end{array}$ & $\begin{array}{l}\text { Telerehabilitation: } \\
35 \text { min }\end{array}$ & $\begin{array}{l}12 \text { weeks, } 12 \\
\text { months }\end{array}$ & $\begin{array}{l}\text { No difference in } 6 \mathrm{MWD} \text { improvement, only } \\
\text { sustained at } 12 \text {-week follow-up in } \\
\text { telerehabilitation group, no difference } \\
\text { between groups at } 12 \text { months }\end{array}$ \\
\hline & & & & & $\begin{array}{l}\text { Hospital-based } \\
\text { rehabilitation: } \\
2 \times / \text { week }\end{array}$ & $\begin{array}{l}\text { Hospital-based } \\
\text { rehabilitation: } \\
60 \text { min }\end{array}$ & & \\
\hline Chaplin et al. (65) & $\begin{array}{l}\mathrm{Y} / \mathrm{COPD} / \mathrm{n}= \\
103\end{array}$ & $\begin{array}{l}\text { Indirect supervision, } \\
\text { web-based program, } \\
\text { telephone; aerobic and } \\
\text { strength training }\end{array}$ & $\begin{array}{l}\text { Up to } 15 \\
\text { weeks }\end{array}$ & $\begin{array}{l}\text { Web-based rehabilitation vs. } \\
\text { conventional rehabilitation }\end{array}$ & $2 \times /$ week & $60 \mathrm{~min}$ & No follow-up & $\begin{array}{l}\text { Similar improvement in exercise capacity } \\
\text { and quality of life; higher dropout rate in } \\
\text { web-based group }\end{array}$ \\
\hline
\end{tabular}


TABLE 2 | Continued

\begin{tabular}{|c|c|c|c|c|c|c|c|c|}
\hline Research study & $\begin{array}{l}\text { RCT/ } \\
\text { Population/ } \\
\text { Sample Size }\end{array}$ & $\begin{array}{l}\text { Mode of delivery/ } \\
\text { exercise }\end{array}$ & $\begin{array}{l}\text { Intervention } \\
\text { duration }\end{array}$ & Comparator groups & $\begin{array}{l}\text { Training } \\
\text { frequency }\end{array}$ & $\begin{array}{l}\text { Training } \\
\text { duration }\end{array}$ & Follow-up & Results \\
\hline Bourne et al. (66) & $\mathrm{Y} / \mathrm{COPD} / n=90$ & $\begin{array}{l}\text { No supervision, web-based } \\
\text { videos; strengthening } \\
\text { exercises }\end{array}$ & 6 weeks & $\begin{array}{l}\text { Online rehabilitation vs. } \\
\text { face-to-face rehabilitation }\end{array}$ & $2-5 \times /$ week & $\begin{array}{l}10 \text { exercises, } \\
30-60 \text { s each }\end{array}$ & No follow-up & $\begin{array}{l}\text { Similar non-significant improvements in } \\
6 M W T \text { and COPD assessment test }\end{array}$ \\
\hline \multirow[t]{2}{*}{$\begin{array}{l}\text { Cameron-Tucker } \\
\text { et al. (67) }\end{array}$} & $\mathrm{Y} / \mathrm{COPD} / n=65$ & $\begin{array}{l}\text { Indirect supervision, } \\
\text { telephone; walking }\end{array}$ & 16-20 weeks & $\begin{array}{l}\text { Telerehabilitation and } \\
\text { outpatient rehabilitation vs. } \\
\text { outpatient rehabilitation } \\
\text { alone }\end{array}$ & $\begin{array}{l}\text { Telerehabilitation: } \\
\text { daily }\end{array}$ & $\begin{array}{l}\text { Telerehabilitation: } \\
30 \text { min }\end{array}$ & No follow-up & No improvement in 6MWD in either group \\
\hline & & & & & $\begin{array}{l}\text { Outpatient } \\
\text { rehabilitation: } \\
2 \times / \text { week }\end{array}$ & $\begin{array}{l}\text { Outpatient } \\
\text { rehabilitation: } 1 \mathrm{~h}\end{array}$ & & \\
\hline \multirow[t]{2}{*}{$\begin{array}{l}\text { Vasilopoulou et al. } \\
\text { (68) }\end{array}$} & $\begin{array}{l}\mathrm{Y} / \mathrm{COPD} / n= \\
147\end{array}$ & $\begin{array}{l}\text { Indirect supervision, } \\
\text { telephone or } \\
\text { videoconference; walking, } \\
\text { arm and leg exercises }\end{array}$ & 12 months & $\begin{array}{l}\text { Outpatient and home } \\
\text { maintenance rehabilitation } \\
\text { vs. outpatient and hospital } \\
\text { maintenance rehabilitation } \\
\text { vs. usual care }\end{array}$ & $\begin{array}{l}\text { Home } \\
\text { maintenance: } 144 \\
\text { total sessions }\end{array}$ & Not reported & No follow-up & $\begin{array}{l}\text { Both home maintenance and hospital } \\
\text { maintenance groups maintained } \\
\text { improvements in } 6 \mathrm{MWT} \text { and peak work } \\
\text { rate, decreased risk of COPD } \\
\text { exacerbations, hospitalizations }\end{array}$ \\
\hline & & & & & $\begin{array}{l}\text { Hospital } \\
\text { maintenance: } \\
2 \times / \text { week }\end{array}$ & & & \\
\hline Tabak et al. (69) & $\mathrm{Y} / \mathrm{COPD} / \mathrm{n}=34$ & $\begin{array}{l}\text { No supervision, smartphone } \\
\text { app and text message; } \\
\text { walking }\end{array}$ & 4 weeks & $\begin{array}{l}\text { Telerehabilitation vs. usual } \\
\text { care }\end{array}$ & Not reported & Not reported & No follow-up & $\begin{array}{l}\text { Improvement in health status in } \\
\text { telerehabilitation group; no change in } \\
\text { activity levels in either group }\end{array}$ \\
\hline Tsai et al. (71) & $\mathrm{Y} / \mathrm{COPD} / \mathrm{n}=37$ & $\begin{array}{l}\text { Direct supervision, webcam; } \\
\text { cycling, walking, } \\
\text { strengthening exercises }\end{array}$ & 8 weeks & $\begin{array}{l}\text { Telerehabilitation vs. } \\
\text { non-exercise control group }\end{array}$ & $3 \times /$ week & Not reported & No follow-up & $\begin{array}{l}\text { Significant increase in exercise capacity in } \\
\text { telerehabilitation group }\end{array}$ \\
\hline Holland et al. (72) & $\mathrm{N} / \mathrm{COPD} / n=8$ & $\begin{array}{l}\text { Direct supervision, webcam; } \\
\text { cycling }\end{array}$ & 8 weeks & $\begin{array}{l}\text { Telerehabilitation only, no } \\
\text { control group }\end{array}$ & $2 \times /$ week & $30 \mathrm{~min}$ & No follow-up & $\begin{array}{l}\text { Significant improvements in 6MWD, } \\
\text { dyspnea, and fatigue; high program } \\
\text { usability and safety }\end{array}$ \\
\hline Zanaboni et al. (73) & $\mathrm{N} / \mathrm{COPD} / n=10$ & $\begin{array}{l}\text { Direct supervision, webcam; } \\
\text { aerobic treadmill exercise, } \\
\text { strength training }\end{array}$ & 2 years & $\begin{array}{l}\text { Telerehabilitation following } \\
\text { outpatient rehabilitation, no } \\
\text { control group }\end{array}$ & $3 \times /$ week & $30 \mathrm{~min}$ & No follow-up & $\begin{array}{l}\text { Maintained 6MWD, lung capacity, health } \\
\text { status, and quality of life, and reduced } \\
\text { healthcare utilization }\end{array}$ \\
\hline
\end{tabular}


TABLE 3 | Characteristics and outcomes of home-based tele-exercise studies - neurological.

\begin{tabular}{|c|c|c|c|c|c|c|c|c|}
\hline Research study & $\begin{array}{l}\text { RCT/ } \\
\text { population/ } \\
\text { sample size }\end{array}$ & Mode of delivery/exercise & $\begin{array}{l}\text { Intervention } \\
\text { duration }\end{array}$ & Comparator groups & $\begin{array}{l}\text { Training } \\
\text { frequency }\end{array}$ & $\begin{array}{l}\text { Training } \\
\text { duration }\end{array}$ & Follow-up & Results \\
\hline $\begin{array}{l}\text { Chumbler et al. } \\
(82,83)\end{array}$ & $\mathrm{Y} /$ Stroke/n $=52$ & $\begin{array}{l}\text { Indirect supervision, telephone and } \\
\text { email; strength and balance exercises }\end{array}$ & 3 months & $\begin{array}{l}\text { Telerehabilitation vs. usual } \\
\text { care (outpatient } \\
\text { rehabilitation) }\end{array}$ & Not reported & Not reported & No follow-up & $\begin{array}{l}\text { No significant differences in physical } \\
\text { function measures (Late-Life Function and } \\
\text { Disability Instrument Function and } \\
\text { Telephone Version of Functional } \\
\text { Independence Measure) or fall-related } \\
\text { self-efficacy in either group }\end{array}$ \\
\hline Chen et al. (84) & Y/Stroke/n = 54 & $\begin{array}{l}\text { Direct supervision, webcam; } \\
\text { occupational and physical therapy } \\
\text { exercises, EMG-triggered } \\
\text { neuromuscular stimulation }\end{array}$ & 12 weeks & $\begin{array}{l}\text { Telerehabilitation vs. } \\
\text { conventional rehabilitation }\end{array}$ & $10 \times /$ week & $60 \mathrm{~min}$ & 12 weeks & $\begin{array}{l}\text { Significant improvements in both groups in } \\
\text { measures of disability and daily living, } \\
\text { balance, and muscular contraction } \\
\text { intensity }\end{array}$ \\
\hline Chen et al. $(85,86)$ & Y/Stroke/n = 52 & $\begin{array}{l}\text { Direct supervision, webcam; } \\
\text { occupational and physical therapy } \\
\text { exercises, EMG-triggered } \\
\text { neuromuscular stimulation }\end{array}$ & 12 weeks & $\begin{array}{l}\text { Telerehabilitation vs. } \\
\text { conventional rehabilitation }\end{array}$ & 10x/week & $80 \mathrm{~min}$ & 12 weeks & $\begin{array}{l}\text { Significant improvements in FMA and } \\
\text { resting-state functional connectivity in both } \\
\text { groups, maintained at } 12 \text {-week follow-up }\end{array}$ \\
\hline \multirow[t]{2}{*}{$\begin{array}{l}\text { Kairy et al. (87), } \\
\text { Norouzi-Gheidari } \\
\text { et al. (88) }\end{array}$} & Y/Stroke/ $n=18$ & $\begin{array}{l}\text { No supervision, Microsoft Kinect; } \\
\text { occupational/physical therapy } \\
\text { exercises, upper extremity exercises } \\
\text { including tracing, reaching, moving, } \\
\text { and clapping }\end{array}$ & 4 weeks & $\begin{array}{l}\text { Usual care and exercise } \\
\text { gaming vs. usual care alone }\end{array}$ & $\begin{array}{l}\text { Usual care: } \\
2-3 \times / \text { week }\end{array}$ & $\begin{array}{l}\text { Usual care: Not } \\
\text { reported }\end{array}$ & No follow-up & $\begin{array}{l}\text { Significant improvements in activities of } \\
\text { daily living measures and mobility and } \\
\text { physical domains of the Stroke Impact } \\
\text { Scale in both groups, greater } \\
\text { (non-significant) improvements with } \\
\text { exercise gaming }\end{array}$ \\
\hline & & & & & $\begin{array}{l}\text { Exercise } \\
\text { gaming: } \\
\text { 2-3x/week }\end{array}$ & $\begin{array}{l}\text { Exercise } \\
\text { gaming: } 30 \mathrm{~min}\end{array}$ & & \\
\hline Lloréns et al. (89) & $\mathrm{Y} /$ Stroke/n $=30$ & $\begin{array}{l}\text { No supervision, Microsoft Kinect; } \\
\text { stepping exercises }\end{array}$ & 8 weeks & $\begin{array}{l}\text { Home-based VR } \\
\text { rehabilitation Vs. } \\
\text { clinic-based VR } \\
\text { rehabilitation training }\end{array}$ & $3 \times /$ week & $45 \mathrm{~min}$ & No follow-up & $\begin{array}{l}\text { Similar clinically meaningful improvements } \\
\text { in gait and balance; lower costs in } \\
\text { home-based group }\end{array}$ \\
\hline Linder et al. (90) & Y/Stroke/n = 99 & $\begin{array}{l}\text { Indirect supervision, telephone; range } \\
\text { of motion, weight-bearing, } \\
\text { active-assistive, activities of daily } \\
\text { living exercises }\end{array}$ & 8 weeks & $\begin{array}{l}\text { Home exercise vs. home } \\
\text { exercise and robot-assisted } \\
\text { therapy }\end{array}$ & $5 \times /$ week & $3 \mathrm{~h}$ & No follow-up & $\begin{array}{l}\text { Significant improvements in quality of life } \\
\text { and depression scales in both groups }\end{array}$ \\
\hline Sarfo et al. (91) & $\mathrm{N} /$ Stroke/n $=20$ & $\begin{array}{l}\text { Indirect supervision, smartphone app } \\
\text { and telephone; mobility, } \\
\text { strengthening, dexterity, balance, and } \\
\text { walking exercises }\end{array}$ & 12 weeks & $\begin{array}{l}\text { Telerehabilitation only, no } \\
\text { control group }\end{array}$ & $5 \times /$ week & $30-60 \mathrm{~min}$ & No follow-up & $\begin{array}{l}\text { Improvements in baseline motor deficits; } \\
\text { high adherence and satisfaction }\end{array}$ \\
\hline Szturm et al. (92) & $\mathrm{N} /$ Stroke $/ n=10$ & $\begin{array}{l}\text { Indirect supervision, video game, } \\
\text { email and telephone; game-assisted } \\
\text { exercises using object manipulation } \\
\text { tasks }\end{array}$ & 16 weeks & $\begin{array}{l}\text { Telerehabilitation only, no } \\
\text { control group }\end{array}$ & $4 \times /$ week & 20-30 min & No follow-up & $\begin{array}{l}\text { Improvements to upper extremity motor } \\
\text { ability; high feasibility and acceptability }\end{array}$ \\
\hline Paul et al. (105) & $\mathrm{Y} / \mathrm{MS} / n=30$ & $\begin{array}{l}\text { Indirect supervision, telephone and } \\
\text { website; aerobic, strengthening, and } \\
\text { balance exercise }\end{array}$ & 12 weeks & $\begin{array}{l}\text { Telerehabilitation vs. usual } \\
\text { care }\end{array}$ & $\begin{array}{l}2 \times / \text { week } \\
\text { (minimum) }\end{array}$ & Not reported & No follow-up & $\begin{array}{l}\text { No significant differences in } 25 \mathrm{ft} \text { walk } \\
\text { between groups or within groups; high } \\
\text { satisfaction }\end{array}$ \\
\hline
\end{tabular}




\begin{tabular}{|c|c|c|c|c|c|c|c|c|}
\hline Research study & $\begin{array}{l}\text { RCT/ } \\
\text { population/ } \\
\text { sample size }\end{array}$ & Mode of delivery/exercise & $\begin{array}{l}\text { Intervention } \\
\text { duration }\end{array}$ & Comparator groups & $\begin{array}{l}\text { Training } \\
\text { frequency }\end{array}$ & $\begin{array}{l}\text { Training } \\
\text { duration }\end{array}$ & Follow-up & Results \\
\hline Paul et al. (106) & $\mathrm{Y} / \mathrm{MS} / n=90$ & $\begin{array}{l}\text { Indirect supervision, telephone and } \\
\text { website; aerobic, strengthening, and } \\
\text { balance exercise }\end{array}$ & 6 months & $\begin{array}{l}\text { Telerehabilitation vs. active } \\
\text { comparator }\end{array}$ & $2 \times /$ week & Not reported & 3 months & $\begin{array}{l}\text { No significant differences in 2-min walk } \\
\text { test or secondary outcomes between } \\
\text { groups or within groups; no difference in } \\
\text { adherence between groups, which } \\
\text { decreased over time }\end{array}$ \\
\hline \multirow[t]{2}{*}{ Tallner et al. (107) } & $\mathrm{Y} / \mathrm{MS} / n=126$ & $\begin{array}{l}\text { Indirect supervision, website, } \\
\text { telephone, email, strength, and } \\
\text { aerobic training }\end{array}$ & 6 months & $\begin{array}{l}\text { Home-based exercise vs. } \\
\text { waitlist control }\end{array}$ & $\begin{array}{l}\text { Strength: } \\
2 \times / \text { week }\end{array}$ & $\begin{array}{l}\text { Strength: } 2-3 \\
\text { sets per exercise }\end{array}$ & No follow-up & $\begin{array}{l}\text { Significant differences between groups in } \\
\text { muscle strength, peak expiratory flow, and } \\
\text { sports activity; high compliance that } \\
\text { decreased over time }\end{array}$ \\
\hline & & & & & $\begin{array}{l}\text { Aerobic: } \\
\text { 1×/week }\end{array}$ & $\begin{array}{l}\text { Aerobic: } \\
\text { 10-60 min }\end{array}$ & & \\
\hline $\begin{array}{l}\text { Keytsman et al. } \\
(108)\end{array}$ & $\mathrm{N} / \mathrm{MS} / \mathrm{n}=45$ & $\begin{array}{l}\text { Direct supervision, telephone and } \\
\text { email; high intensity cycling }\end{array}$ & 6 months & $\begin{array}{l}\text { Persons with MS vs. healthy } \\
\text { controls }\end{array}$ & $3 \times /$ week & $\begin{array}{l}1-3 \mathrm{~h} \text { and } \\
60-90 \mathrm{~s} \text { of } \\
\text { maximal interval } \\
\text { training }\end{array}$ & No follow-up & $\begin{array}{l}\text { Similar significant improvements in peak } \\
\mathrm{VO}_{2} \text {, reductions in body mass }\end{array}$ \\
\hline $\begin{array}{l}\text { Finkelstein et al. } \\
\text { (109) }\end{array}$ & $\mathrm{N} / \mathrm{MS} / \mathrm{n}=12$ & $\begin{array}{l}\text { Indirect supervision, telephone and } \\
\text { computerized system; functional } \\
\text { strength, stretching, and balance } \\
\text { exercises }\end{array}$ & 12 weeks & $\begin{array}{l}\text { Telerehabilitation only, no } \\
\text { control group }\end{array}$ & $\begin{array}{l}\text { Customized } \\
\text { to participant }\end{array}$ & $\begin{array}{l}\text { Customized to } \\
\text { participant }\end{array}$ & No follow-up & $\begin{array}{l}\text { Significant improvements in } 25 \mathrm{ft} \text { walk, } \\
6 \mathrm{MWD} \text {, and balance; high satisfaction }\end{array}$ \\
\hline \multirow[t]{2}{*}{$\begin{array}{l}\text { Fjeldstad-Pardo } \\
\text { et al. (110) }\end{array}$} & $\mathrm{Y} / \mathrm{MS} / n=30$ & $\begin{array}{l}\text { Direct supervision, webcam; physical } \\
\text { therapy exercises }\end{array}$ & 8 weeks & $\begin{array}{l}\text { Supervised telerehabilitation } \\
\text { vs. unsupervised home } \\
\text { exercise vs. in-person } \\
\text { rehabilitation }\end{array}$ & \multicolumn{2}{|c|}{$\begin{array}{l}\text { TelerehabilitatiołNot reported } \\
\text { and in-person } \\
\text { rehabilitation: } \\
2 \times / \text { week }\end{array}$} & No follow-up & $\begin{array}{l}\text { Significant improvements in gait and } \\
\text { balance in all groups, similar } \\
\text { improvements between telerehabilitation } \\
\text { and in-person rehabilitation groups }\end{array}$ \\
\hline & & & & & $\begin{array}{l}\text { Home } \\
\text { exercise: } \\
\text { 5x/week }\end{array}$ & & & \\
\hline $\begin{array}{l}\text { van der Kolk et al. } \\
\text { (117) }\end{array}$ & $\mathrm{N} / \mathrm{PD} / \mathrm{n}=37$ & $\begin{array}{l}\text { Indirect supervision, telephone, tablet } \\
\text { app, web-based videos; cycling }\end{array}$ & 6 months & $\begin{array}{l}\text { Home-based exercise vs. } \\
\text { usual care }\end{array}$ & $3-5 \times /$ week & $45 \mathrm{~min}$ & No follow-up & $\begin{array}{l}\text { Significant improvement in peak } \mathrm{VO}_{2} \text { in } \\
\text { home-based group, high adherence and } \\
\text { low dropout rate }\end{array}$ \\
\hline $\begin{array}{l}\text { van der Kolk et al. } \\
\text { (118) }\end{array}$ & $\mathrm{Y} / \mathrm{PD} / \mathrm{n}=130$ & $\begin{array}{l}\text { Indirect supervision, telephone, tablet } \\
\text { app, web-based videos; cycling }\end{array}$ & 6 months & $\begin{array}{l}\text { Home-based exercise vs. } \\
\text { active control (stretching) }\end{array}$ & $3 \times /$ week & $30-45$ min & No follow-up & $\begin{array}{l}\text { Significant improvements in peak } \mathrm{VO}_{2} \text { and } \\
\text { PD symptoms in home-based group }\end{array}$ \\
\hline $\begin{array}{l}\text { Gandolfi et al. } \\
\text { (119) }\end{array}$ & $\mathrm{Y} / \mathrm{PD} / \mathrm{n}=76$ & $\begin{array}{l}\text { Direct supervision, webcam and } \\
\text { Nintendo Wii; exercise games, } \\
\text { balance exercises }\end{array}$ & 7 weeks & $\begin{array}{l}\text { VR telerehabilitation vs. } \\
\text { clinic-based balance training }\end{array}$ & $3 \times /$ week & $50 \mathrm{~min}$ & 1 month & $\begin{array}{l}\text { Statistically greater improvements in } \\
\text { mobility, balance for clinic group, lower } \\
\text { cost in telerehabilitation group }\end{array}$ \\
\hline Lai et al. (120) & $\mathrm{N} / \mathrm{PD} / n=20$ & $\begin{array}{l}\text { Direct supervision, webcam; strength } \\
\text { and aerobic exercise }\end{array}$ & 8 weeks & $\begin{array}{l}\text { Telecoach-assisted exercise } \\
\text { vs. self-regulated exercise }\end{array}$ & $3 \times /$ week & $\begin{array}{l}20-55 \text { min, } \\
\text { progressively } \\
\text { increasing by } \\
\text { week }\end{array}$ & No follow-up & $\begin{array}{l}\text { Small to moderate improvements in } 6 \mathrm{MWT} \\
\text { in telecoach group, higher attendance and } \\
\text { time spent exercising in telecoach group }\end{array}$ \\
\hline Seidler et al. (121) & $\mathrm{N} / \mathrm{PD} / \mathrm{n}=26$ & $\begin{array}{l}\text { Direct supervision, webcam; tango } \\
\text { dance }\end{array}$ & 12 weeks & $\begin{array}{l}\text { Telerehabilitation vs. } \\
\text { in-person instruction }\end{array}$ & $2 \times /$ week & $60 \mathrm{~min}$ & No follow-up & $\begin{array}{l}\text { Similar significant improvements in } \\
\text { balance and motor sign severity, } \\
\text { comparable retention and attendance } \\
\text { rates between groups }\end{array}$ \\
\hline Lai et al. (125) & $\mathrm{N} / \mathrm{SCl} / n=4$ & $\begin{array}{l}\text { Direct supervision, webcam; aerobic } \\
\text { exercise (upper body ergometer) }\end{array}$ & 8 weeks & $\begin{array}{l}\text { Teleexercise only, no control } \\
\text { group }\end{array}$ & $3 \times /$ week & $30-45$ min & No follow-up & $\begin{array}{l}\text { Improvement in peak } \mathrm{VO}_{2} \text {, increased daily } \\
\text { physical activity, high adherence }\end{array}$ \\
\hline
\end{tabular}




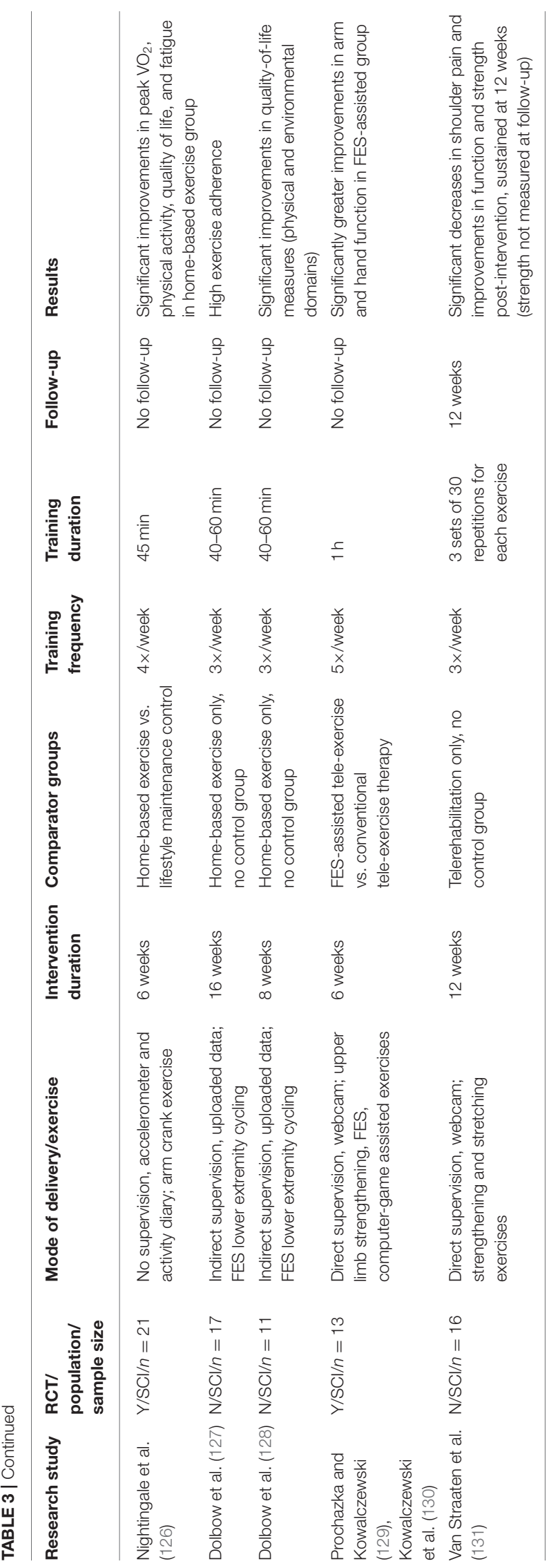

strengthening exercises, range of motion exercises, and flexibility exercises $(21,22)$. One RCT of individuals with knee OA was done using indirect supervision via weekly telephone calls, with exercises being done to improve strength, endurance, flexibility, and range of motion. Compared to the clinicbased group, those receiving telerehabilitation had similar significant improvements in the Knee Injury and Osteoarthritis Outcome Score and the WOMAC (22). Follow-up assessments were performed 1 and 6 months post-treatment, and no significant differences in improvement were seen between groups. Another study of individuals with knee OA was done using directly supervised exercise, assessing qualitative outcomes regarding participants' experience of the program (21). Only a telerehabilitation group was assessed during the study, with participants expressing high satisfaction with the program, finding it convenient, and appreciating not having to travel to a clinic. Participants also reported reductions in knee pain as well as improvements in physical function, allowing them to be more active (21). Two studies using telerehabilitation exercises have been done for individuals receiving knee or hip replacements, with both using indirect supervision (2325). Both studies showed significant improvements in functional measures including the timed up-and-go and 6-min walk time (6MWT), and significant improvements in WOMAC scores. For one of the studies, these improvements were similar between the telerehabilitation group and inpatient rehabilitation control group (25), while the other study was only a pilot study in which no control group was used (23). In a telerehabilitation study of individuals with injuries to lower extremities, a novel smartphone application was created that used equipped sensors that could track movement and walking activity (26). For 3 months, participants in the telerehabilitation group performed home-based exercises with passive and active flexion-extension and dosed load on the injured limb, with the smartphone attached to the leg. Outcomes measured for this study were patient satisfaction with rehabilitation as well as time spent in consultation with their orthopedic surgeon. Compared to a control group who received traditional methods of rehabilitation such as massage, myostimulation, and pool exercises, participants in the telerehabilitation group had higher satisfaction with the intervention, as well as less time spent per visit time with their orthopedic surgeon (26). Overall, these studies utilizing home-based exercise programs for rehabilitation of the lower extremities have had varying lengths, but have generally produced significant functional improvements and high satisfaction. When long-term follow ups have been performed, improvements have persisted, granted in a limited number of studies. As improvements have been comparable to traditional exercise rehabilitation therapies, these studies show home-based telerehabilitation is a viable option for those unable or unwilling to travel for in-person services.

Home-based exercise has also been used to remedy injuries of the upper extremity, particularly for ailments pertaining to the shoulder. RCTs using home-based rehabilitation exercises have been conducted with no supervision for conditions and ailments such as arthroscopic subacromial decompression (ASD) surgery $(27,28)$, frozen shoulder (29), and chronic shoulder 
pain (30). In these studies, home-based rehabilitation resulted in significant improvements in pain and function similar to conventional rehabilitation $(28,29)$ or greater than education only (30). One pilot study for individuals with rotator cuff pain was designed to compare the feasibility of three different internetbased interventions including advice only, recommended care, and recommended care with telerehabilitation. As this was a feasibility study, the primary outcomes were adherence, retention, and number of adverse events. Acceptable exercise adherence was seen for the telerehabilitation group only, while incidence rates of adverse events were similar between all three groups (31). In a study of individuals who recently had shoulder replacement surgery, a directly supervised telerehabilitation program was compared to a control group receiving inperson physiotherapy. Significant improvements in pain and shoulder function ability were observed in both groups; however, the telerehabilitation group experienced significantly greater improvements compared to those of the control group (32). Interviews with the participants in the telerehabilitation group revealed a positive experience with the intervention, due to the ability to still be able to interact with the physiotherapist one-on-one while performing the exercises in their own homes (33). For individuals suffering from a fracture to the proximal humerus, a pilot study consisting of a program of stretching, pain control, active range of motion, and muscle building exercise was performed to determine its feasibility. After rehabilitation, a significant decrease in self-reported pain was observed along with a significant improvement in range of motion measurements including flexion, extension, and rotation. Additionally, upper limb function measured by the Disability of the Arm, Shoulder and Hand questionnaire showed significant, clinically relevant improvement. Participants reported high overall satisfaction with the intervention regarding the services they received (34). One more pilot study of shoulder rehabilitation has examined the feasibility of a Microsoft Kinect-based telerehabilitation system where participants performed physiotherapeutic exercises. All participants showed relative improvement in shoulder range of motion and reported high useability and satisfaction with the program (35). Similar to studies of the lower extremities, homebased exercises for rehabilitation of upper extremity ailments have shown to be effective in inducing functional improvements over various study durations. In studies that have compared telerehabilitation to conventional rehabilitation, no differences in improvements have been seen. Additionally, pilot and feasibility studies of telerehabilitation in individuals with upper extremity ailments have been generally well-received, with high satisfaction and improvements in measured functional outcomes.

\section{CARDIORESPIRATORY}

\section{Cardiovascular Disease}

Following an acute cardiac event, cardiac rehabilitation (CR) provides a therapy for recovery, in which a structured, hospitalbased exercise prescription is given along with patient education and risk factor modification (36). While exercise prescription can vary due to each patient's functional capacity and overall condition, programs generally contain weekly, aerobic exercise sessions that are targeted to a percentage of the patient's peak heart rate and are progressively increased in intensity, dependent on tolerance (37). Although hospital-based cardiac rehabilitation has been a long-established method of therapy, home-based cardiac rehabilitation is becoming an option for individuals with limited mobility or transportation, increasing participation and access to care (38).

Studies of home-based CR have compared its efficacy to conventional CR, using both aerobic exercise alone (39-43) or a combination of aerobic and strength training exercises (4446). These RCTs yielded positive results, independent of the degree to which participants were supervised during exercise, with similar improvements in outcomes such as peak oxygen consumption $\left(\mathrm{VO}_{2}\right)(40,43), 6$-min walk distance (6MWD) (44), and exercise time and capacity (46). Of the studies that performed follow-up assessments, no differences between the home-based CR groups or conventional CR groups were observed at 12 weeks $(40,44)$ or 1 year $(43)$ following the completion of CR. Two other RCTs have compared home-based CR to usual care without a dedicated exercise component, yielding mixed results. One of the studies utilized indirectly supervised walking or jogging in the home-based CR group, which resulted in significantly greater improvements in 6MWT and quality of life measures compared to usual care (47). In the other study, participants in the home-based CR group received weekly text messages encouraging them to engage in moderate to vigorous aerobic activity, but were not directly supervised by researchers (48). While the intervention was engaging and positively received (49), participants in the intervention group and control group had no differences in exercise capacity measured by peak $\mathrm{VO}_{2}$ following the program. The authors believe this may be due to insufficient intensity of exercise due to participants' perception of moderate to vigorous exercise. However, those in the intervention group did have significant increases in leisure time physical activity and walking, as well as self-efficacy to be active $(50,51)$. In one other RCT of telerehabilitation, participants were given center-based rehabilitation focused on diet, wellbeing, behavior, and exercise training followed by a period of home-based CR, or no additional intervention. Additionally, those in the home-based CR group also received the telerehabilitation intervention during the last 6 weeks of their center-based CR. Home-based CR consisted of the use of an accelerometer and a semiautomatic coaching system which provided weekly feedback on their performance via email or text message (52). Significant improvements in peak $\mathrm{VO}_{2}$ were observed for the telerehabilitation group following the end of the 6-month program but returned to baseline values at 2-year follow-up evaluations. However, participants who only received the center-based rehabilitation program had a progressive decrease in peak $\mathrm{VO}_{2}$ at the end of the 6-month program and at 2-year follow up, with a significantly lower peak $\mathrm{VO}_{2}$ at 2-year follow up compared to the tele-exercise group. Also, the average cost per patient was $\sim 1,000$ dollars lower in the intervention group compared to the control group over the course of the program (53). Nearly all studies in which $\mathrm{CR}$ was delivered with an exercise component were found to improve physical outcomes following the rehabilitation program. These improvements when using telerehabilitation methods were 
similar to those of conventional rehabilitation, or superior to usual care without an exercise intervention. While both short and long-term telerehabilitation programs had positive outcomes, all of the studies consisted of an aerobic exercise component, which would support its importance for effective CR.

\section{Chronic Obstructive Pulmonary Disease}

Individuals with chronic obstructive pulmonary disease (COPD) can undergo pulmonary rehabilitation (PR) to improve lung function, which often includes an exercise training component. This exercise training has shown to be able to reduce dyspnea and fatigue, as well as improve quality of life and exercise tolerance (54). Modalities of exercise that have been shown to be beneficial to those with COPD include aerobic exercise training $(55,56)$, resistance training (57), interval training (58), and inspiratory muscle training (59). While these exercise programs are typically performed in a clinical setting, studies using exercise training programs designed for use at home or remotely have also been employed.

Multiple RCTs have been conducted in individuals with COPD, comparing telerehabilitation vs. conventional PR methods. These studies have used differing exercise modalities, including aerobic exercise $(60,61)$, aerobic exercise and strength training (62-65), and strength training alone (66). No significant difference in improvements were observed between groups for outcomes including $6 \mathrm{MWD}(61,63,66)$, exercise capacity (65), and quality of life (65). However, in the studies that performed long-term follow-ups, these improvements were lost in both groups following 1 year $(61,64)$. Two RCTs have also been conducted comparing telerehabilitation plus conventional PR vs. conventional PR alone. In one of the RCTs, participants in the telerehabilitation group established a home-based walking plan, with weekly telephone calls with nurse mentors, while those in the control group were not instructed or contacted by researchers. Following this 8-week period, both groups then underwent conventional, center-based PR. Neither group had significant improvements in 6MWD or secondary outcomes following either the initial 8 week period, or 8-12 weeks of conventional PR (67). In the other RCT using a combination of conventional $\mathrm{PR}$ and telerehabilitation, the effectiveness of an indirectly supervised, home-based telerehabilitation maintenance program was compared to a similar hospitalbased PR maintenance program following conventional PR. Additionally, both groups were compared to a usual care group that did not receive $P R$ and did not have a PR maintenance program. Both the home-based maintenance group and hospital-based maintenance group showed improvements in $6 \mathrm{MWT}$ and peak work rate following their initial PR, and these improvements were maintained following their respective maintenance programs. Also, both groups had decreased risk of COPD exacerbations and hospitalizations compared to the usual care group (68). While the effectiveness of improving outcomes was mixed in these studies of telerehabilitation of COPD patients, improvements or lack thereof were at least similar to those receiving conventional PR. Thus, when designing telerehabilitation programs for individuals with COPD, it is critical to use exercise methods that are well-established to be sure that any potential negative or null outcome is not due to insufficient exercise intensity.

Home-based PR in COPD patients has also been compared to usual care alone, with two RCTs showing generally positive results. Aerobic exercise was used in one of the RCTs, with an unsupervised home-based walking program compared to usual care alone (69). While activity levels did not significantly differ between groups, health status (including clinical status of the airways, activity limitation, emotional dysfunction) measured by the Clinical COPD Questionnaire (70) did improve in the intervention group (69). Another RCT of COPD patients comparing home-based $\mathrm{PR}$ vs. usual care utilized a program of both directly supervised aerobic and strengthening exercises. Following the intervention, those receiving homebased PR had significant increases in exercise capacity and self-efficacy compared to the non-exercising control group (71). While multiple studies have been conducted comparing telerehabilitation vs. conventional PR in COPD patients, two studies of telerehabilitation have been done without comparison to a control group. In a small feasibility study of homebased PR, participants with COPD underwent a cycling program supervised by a physiotherapist. Results showed high useability and safety of the program along with significant improvements in 6MWD, dyspnea, and fatigue (72). In a pilot study of COPD patients who had completed conventional PR, participants took part in a 2-year telerehabilitation maintenance program consisting of both directly supervised aerobic and strength training exercise. Following the program, participants maintained baseline levels in outcomes such as 6MWD, lung capacity, health status, and quality of life (73). In general, these studies of telerehabilitation in individuals with COPD that did not make comparisons to conventional PR showed improvements in measured outcomes from baseline. These findings, along with the findings of studies comparing telerehabilitation to conventional PR, show that telerehabilitation is a viable option for $\mathrm{PR}$ in individuals with COPD.

\section{NEUROLOGICAL}

\section{Stroke}

Following stroke, exercise training is recommended to improve physical health as well as risk management for secondary prevention of stroke (74). With exercise, stroke patients have shown increases in both cardiovascular fitness (75) and muscle strength $(76,77)$. Additionally, exercise may improve walking ability (78), quality of life (79), and fatigue (80) in these individuals. Although there are many potential benefits to exercise following stroke, long-term adherence to a training program has been relatively poor, with up to $50 \%$ of individuals stopping their program within the 1st year (81). More recently, studies of home-based exercise programs have been examined in stroke patients, which may offer an alternative, acceptable method of training.

Multiple RCTs have been conducted in stroke patients using varying types of home-based exercise interventions for rehabilitation purposes. In a RCT of adults who had previously had an ischemic or hemorrhagic stroke in the past 
2 years, participants took part in a telerehabilitation program using indirectly supervised strength and balance exercises $(82,83)$. No significant differences were observed between the telerehabilitation group and the usual care group for primary outcome measures of physical function [motor subscale of the Telephone Version of the Functional Independence Measure (FONEFIM) and the Overall Function Component of the Late-Life Function and Disability Instrument (LLFDI)] or fall-related self-efficacy $(82,83)$. However, there was a significant treatment effect in the telerehabilitation group for the LLFDI Disability Component, a secondary outcome measure representing clinically meaningful improvement on participants' ability to perform life tasks (82). Another RCT of stroke rehabilitation has used a combination of occupational and physical therapy exercises along with EMG-triggered neuromuscular stimulation under direct supervision. Compared to individuals who received the same rehabilitation procedures in an outpatient setting, both groups had similar improvements in measures of disability and daily living, balance, and muscular contraction (84). A later RCT by the same research group using the same patient population and intervention assessed motor function and functional connectivity of the motor cortex areas of the brain. Following the intervention, both the telerehabilitation group and conventional rehabilitation group exhibited significant improvements in measures of motor function and resting-state functional connectivity between the bilateral primary motor cortex areas $(85,86)$. Two RCTs of stroke patients have used virtual reality (VR) platforms, specifically Microsoft Kinect, as a form of telerehabilitation. In one of the studies, a VR exercise gaming program without supervision along with conventional therapy was compared to conventional therapy alone $(87,88)$. Both interventions yielded significant improvements in activities of daily living measures and both mobility and physical domains of the Stroke Impact Scale, with greater (non-significant) improvements in the exercise gaming group (88). Stepping exercise was used as part of another VR rehabilitation program with Microsoft Kinect, with stroke patients using the system at home without supervision or in the clinic. While both groups had similar, meaningful improvements in gait and balance, those in the home-based group had lower costs of therapy (89). One more RCT in stroke patients using indirectly supervised home-exercise had participants receive either home-exercise along with robotassisted therapy or home-exercise alone. Both groups exhibited similar significant improvements of quality of life and depression scales (90).

In addition to the RCTs using telerehabilitation that have been conducted in stroke patients, smaller pilot studies have been performed without the use of a control group. A combination of strength, balance, and walking exercise was used in a pilot study where telerehabilitation was delivered via a smartphone application. Following the intervention, participants had improvements in baseline motor deficits, along with high adherence and satisfaction with the program (91). One other pilot study of telerehabilitation in stroke patients utilized an indirectly supervised, novel game-assisted exercise program focused on object manipulation tasks. Participants in the study displayed improvements to upper extremity mobility, and the program was found to have both high feasibility and acceptability (92). Although varying exercise modalities have been employed in studies of stroke patients undergoing homebase exercise, these training programs have been relatively successful in improvements in functional deficits. However, as long-term follow ups were not performed, it is unknown if these functional improvements can be sustained after the completion of the prescribed exercise programs. Compliance to exercise prescription after clinic-based stroke rehabilitation has been inconsistent and less than ideal $(93,94)$. Thus, it is necessary that future studies in stroke patients utilizing tele-exercise programs also consider long-term follow-up and behavioral factors that will improve adherence to exercise rehabilitation.

\section{Multiple Sclerosis}

A large number of studies examining the effects of exercise training on individuals with multiple sclerosis (MS) have been discussed in other systematic reviews $(95,96)$. MS is a disease of the central nervous system where progressive neurodegeneration results in neurological disability, impaired mobility, and compromised quality of life (97). Exercise training can be an effective tool to manage the functional deficits that occur with MS, helping to manage symptoms of the disease $(98,99)$ as well as improve walking ability $(100,101)$, balance (102), and fatigue $(103,104)$.

Studies that have examined tele-exercise interventions in individuals with MS have used a variety of methods, including aerobic training (105-108), strength training (105-107, 109), balance exercises $(105,106)$, and physical therapy exercises (110). A pilot study of home-based telerehabilitation in individuals with MS used an indirectly supervised program of strength, stretching, and balance exercises customized to each participant, and found significant improvements in outcomes such as $25 \mathrm{ft}$ walk time, $6 \mathrm{MWD}$, and balance. However, as this was a pilot study, comparisons were not made to a control group (109). A similar pilot study of telerehabilitation in individuals with MS used an indirectly supervised program of strength and balance exercises along with aerobic exercise. In this study, neither the telerehabilitation group nor a usual care group displayed changes to $25 \mathrm{ft}$ walk time, but high satisfaction was reported with the telerehabilitation intervention (105). The authors followed this pilot study with a larger RCT using the same methods, but again found no significant differences between groups in primary walking and balance outcomes or secondary outcomes (106). Adherence was high among the telerehabilitation group, but decreased over time (106). A RCT in individuals with MS that focused on strength training with some aerobic training compared a home-based exercise program to a waitlist control group. Significant differences between groups was found for muscle strength, as well as for measures of lung function and physical activity (107). One directly monitored study in individuals with MS utilized a home-based, high intensity cycling program with the intention of improving fitness, exercise capacity, and body composition. Participants with MS and healthy controls had similar significant improvements in peak $\mathrm{VO}_{2}$, while significant reductions in body mass were observed 
only in individuals with MS (108). One more RCT in individuals with MS compared a supervised telerehabilitation program to both an in-person rehabilitation program and an un-supervised home-based exercise program acting as a control group. While all groups exhibited significant improvements in gait and balance measures, improvements were greater in the supervised telerehabilitation and in-person rehabilitation groups compared to the home-exercise group (110). Most of these studies using tele-exercise interventions had positive outcomes following the programs, but did not make comparisons to clinic-based or conventional exercise programs. So, while results of these studies are promising for treating MS symptoms or improving physical function, conclusions cannot be made whether tele-exercise is inferior or comparable to more established exercise programs in this population.

\section{Parkinson's Disease}

Parkinson's disease (PD) is a progressive neurodegenerative disease that presents with a number of functional impairments including postural instability (111), balance problems (112), and gait issues $(113,114)$. In addition to usual therapies including medication and surgical treatments, exercise training may be a complementary option in alleviating symptoms of the disease (115). Types of exercise training that may improve health in individuals with PD include aerobic exercise, gait training, balance training, resistance training, yoga, and dance (116).

Different modalities of tele-exercise with both indirect and direct supervision have been tested in individuals with PD with positive results. A feasibility study of indirectly supervised aerobic cycling exercise was designed for adults with mild PD where participants exercised at home with virtual reality software and real-life videos and had remote coaching. Compared to participants only receiving usual care, participants in the teleexercise group had significant improvements in peak $\mathrm{VO}_{2}$ along with high adherence and low dropout rate (117). A larger follow-up study using the same exercise intervention vs. an active stretching control group yielded similar results, with significant improvements in peak $\mathrm{VO}_{2}$ and $\mathrm{PD}$ symptoms only in the tele-exercise group (118). VR training has also been used as a form of telerehabilitation in individuals with PD. In a RCT using VR exercise, participants in the telerehabilitation group used Nintendo Wii to perform various mobility and balance exercises under direct supervision, while the control group received in-clinic rehabilitation using sensory integration balance training. Significant improvements in balance, gait speed, ability to modify gait, and quality of life were observed in both groups, with improvements in mobility and dynamic balance statistically greater for the in-clinic group (119). However, the practical relevance of these differences compared to the telerehabilitation group were minimal. Satisfaction of the intervention was comparable between groups, but costs were significantly lower in the telerehabilitation group (119). In a pilot study of home-based exercise in PD patients, a program of aerobic and strength training exercise was compared between a group receiving telecoaching and a group self-regulating their exercise. While all participants in the telecoach group exhibited small to moderate improvements in walking capacity, changes to walking capacity in the self-regulated group varied, with some participants decreasing. Additionally, participants in the telecoach group had higher attendance and spent more time exercising than participants in the self-regulated group (120). One more study of tele-exercise in PD patients has used group tango dance as a form of telerehabilitation. Compared to individuals receiving in-person instruction, participants in the telerehabilitation group had similar significant improvements in balance and motor sign severity. Also, attendance rates and retention were comparable between the telerehabilitation and in-person groups (121). Although there have been a limited number of studies utilizing tele-exercise in individuals with PD, the studies conducted had high participation, with comparable improvements in physical function. As the few studies have used different exercise modalities, there are many options for future research that may be viable for managing the symptoms of PD.

\section{Spinal Cord Injury}

Because spinal cord injury (SCI) can occur along different segments of the spine, functional impairment can vary greatly, dependent on the site of injury. Depending on the type of exercise training, exercise can promote functional recovery by helping to strengthen muscles and promote motor function recovery, as well as improve aerobic capacity (122). Various modes of exercise may be utilized by persons with SCI, including electrically stimulated cycling, electrically stimulated ambulation, arm and wheelchair ergometry, resistance training, and respiratory muscle training $(123,124)$. Most studies involving exercise training and rehabilitation in SCI individuals have been performed in a clinical setting, but only a small number have employed tele-exercise.

For individuals with SCI, exercises used in telerehabilitation interventions have varied, including upper extremity cycling $(125,126)$, functional electronic stimulation (FES) lower body cycling $(127,128)$, and upper extremity strengthening exercises (129-131). Many of these studies have been small pilot or feasibility studies, consisting of only a tele-exercise group. The results of these studies have been generally positive, with improvements in outcomes such as shoulder pain, function, and strength (131), peak $\mathrm{VO}_{2}$ (125), and quality-of-life measures (128), along with high exercise adherence $(125,127)$. One RCT has compared two groups of individuals with SCI both receiving directly supervised tele-exercise, with one group using conventional therapy and the other group using FES-assisted therapy. While both groups had significant improvements in arm and hand functions, improvements were statistically greater in the FES-assisted group $(129,130)$. One other RCT in individuals with SCI has compared the use of unsupervised home-based arm crank exercise to a lifestyle maintenance control group. Outcomes that were significantly improved in the intervention group include peak $\mathrm{VO}_{2}$, physical activity levels, quality of life measures, and fatigue, while no significant changes were observed in the control group (126). Individuals with SCI face many barriers to exercise, including lack of accessibility to facilities and traveling restrictions (132). While the few studies using tele-exercise programs in SCI individuals have generally resulted in positive outcomes and have been well-received, 
sample sizes for these studies are relatively small. Future teleexercise studies in this population are needed with a larger number of participants to verify the clinical usability of these exercise programs. As most of the tele-exercise studies performed with SCI individuals lasted 8 weeks or less, longer studies will be needed to determine feasibility of the interventions and whether the positive effects of the interventions are long lasting.

\section{CONCLUSION}

Although exercise training is a vital component in the treatment of many diseases and conditions, ensuring compliance to programs can be an arduous task for clinicians. Individuals participating in these exercise training programs may lack the means to attend in-person sessions or may not want to commit time to traveling to a clinic or gym. As such, remote, tele-exercise training is showing to be a viable, alternative option. Tele-exercise studies using both direct or indirect supervision with the use of webcams, telephone calls, videos, and smartphone applications have produced comparable improvements in outcomes compared to clinic or hospital-based, in-person control interventions. In diseases or conditions where impaired mobility may hinder participation in exercise training programs, these individuals now have the opportunity to undergo exercise training in the home environment. Tele-exercise training programs can be as effective as conventional exercise training without the burden of additional travel. When measured, adherence to tele-exercise programs has been generally high, with the interventions being well-received by participants. Studies

\section{REFERENCES}

1. Thorpe O, Johnston K, Kumar S. Barriers and enablers to physical activity participation in patients with COPD. J Cardiopulm Rehabil Prev. (2012) 32:359-69. doi: 10.1097/HCR.0b013e318262d7df

2. Scelza WM, Kalpakjian CZ, Zemper ED, Tate DG. Perceived barriers to exercise in people with spinal cord injury. Am J Phys Med Rehabil. (2005) 84:576-83. doi: 10.1097/01.phm.0000171172.96290.67

3. Ellis T, Boudreau JK, DeAngelis TR, Brown LE, Cavanaugh JT, Earhart GM, et al. Barriers to exercise in people with Parkinson's disease. Phys Ther. (2013) 93:628-36. doi: 10.2522/ptj.20120279

4. Rimmer JH. Barriers associated with exercise and community access for individuals with stroke. J Rehabil Res Dev. (2008) 45:315-22. doi: 10.1682/JRRD.2007.02.0042

5. Stroud N, Minahan C, Sabapathy S. The perceived benefits and barriers to exercise participation in persons with multiple sclerosis. Disabil Rehabil. (2009) 31:2216-22. doi: 10.3109/09638280902980928

6. Sechrist KR, Walker SN, Pender NJ. Development and psychometric evaluation of the exercise benefits/barriers scale. Res Nurs Health. (1987) 10:357-65. doi: 10.1002/nur.4770100603

7. Hafez AR, Alenazi MA, Kachanathu SJ, Alroumi AM, Mohamed ES. Knee osteoarthritis : a review of literature. Phys Med Rehabil Int. (2014) 1:8.

8. Pandya J, Johnson T, Low AK. Shoulder replacement for osteoarthritis: a review of surgical management. Maturitas. (2018) 108:71-76. doi: 10.1016/j.maturitas.2017.11.013

9. Pozzi F, Snyder-Mackler L, Zeni J. Physical exercise after knee arthroplasty: a systematic review of controlled trials. Eur $J$ Phys Rehabil Med. (2013) 49:877-92. of tele-exercise interventions have ranged in duration from 4 weeks to 2 years in length, with most taking part over the course of 6-12 weeks. These studies have been effective in inducing changes to measured outcomes, but many lack a longterm follow-up to determine if these changes persist. In the studies that did perform long-term follow-up, sustainability of the changes resulting from the interventions varied. Thus, more studies utilizing tele-exercise training need to include long-term follow-ups, as sustained adherence to exercise training programs can be difficult to achieve. Regardless, tele-exercise training offers a promising tool for improving health and well-being and is becoming more viable as advancements in technology continue to progress.

\section{AUTHOR CONTRIBUTIONS}

AA contributed to writing original draft preparation. AR contributed to writing review and editing. AA and AR did conceptualization. Both authors approved the submitted version and final proof of the manuscript.

\section{FUNDING}

This research was supported through funds by a Senior Research Career Scientist Award (ASR) from the United States Department of Veterans Affairs (VA) Rehabilitation R\&D (Rehab RD) Service, VA Merit Award 001461(ASR) RR\&D, VA Medical Center Baltimore Geriatric Research, Education and Clinical Center (GRECC), and National Institutes of Health Grant P30-AG028747.

10. Dávila Castrodad IM, Recai TM, Abraham MM, Etcheson JI, Mohamed NS, Edalatpour A, et al. Rehabilitation protocols following total knee arthroplasty: a review of study designs and outcome measures. Ann Transl Med. (2019) 7:S255-S5. doi: 10.21037/atm.2019.08.15

11. Doiron-Cadrin P, Lafrance S, Saulnier M, Cournoyer É, Roy J-S, Dyer J$\mathrm{O}$, et al. Shoulder rotator cuff disorders: a systematic review of clinical practice guidelines and semantic analyses of recommendations. Arch Phys Med Rehabil. (2020) 101:1233-42. doi: 10.1016/j.apmr.2019.12.017

12. Thigpen CA, Shaffer MA, Gaunt BW, Leggin BG, Williams GR, Wilcox RB. The American Society of Shoulder and Elbow Therapists' consensus statement on rehabilitation following arthroscopic rotator cuff repair. J Shoulder Elb Surg. (2016) 25:521-35. doi: 10.1016/j.jse.2015. 12.018

13. Bandholm T, Wainwright TW, Kehlet H. Rehabilitation strategies for optimisation of functional recovery after major joint replacement. $J$ Exp Orthop. (2018) 5:44. doi: 10.1186/s40634-018-0156-2

14. Ben KW. Shoulder rehabilitation: principles and practice. Med Sci Sport Exerc. (1998) 30:40-50. doi: 10.1097/00005768-19980400 1-00007

15. Russell TG, Buttrum P, Wootton R, Jull GA. Internet-based outpatient telerehabilitation for patients following total knee arthroplasty. J Bone Jt Surg Am. (2011) 93:113-120. doi: 10.2106/JBJS.I.01375

16. Tousignant $\mathrm{M}$, Moffet $\mathrm{H}$, Boissy $\mathrm{P}$, Corriveau $\mathrm{H}$, Cabana F, Marquis F. A randomized controlled trial of home telerehabilitation for post-knee arthroplasty. J Telemed Telecare. (2011) 17:1958. doi: 10.1258/jtt.2010.100602

17. Moffet H, Tousignant M, Nadeau S, Mérette C, Boissy P, Corriveau H, et al. In-home telerehabilitation compared with face-to-face rehabilitation 
after total knee arthroplasty. J Bone Jt Surg Am. (2015) 97:112941. doi: 10.2106/JBJS.N.01066

18. Tousignant M, Moffet H, Nadeau S, Mérette C, Boissy P, Corriveau H, et al. Cost analysis of in-home telerehabilitation for post-knee arthroplasty. J Med Internet Res. (2015) 17:e83. doi: 10.2196/jmir.3844

19. Prvu Bettger J, Green CL, Holmes DN, Chokshi A, Mather RC, Hoch BT, et al. Effects of virtual exercise rehabilitation in-home therapy compared with traditional care after total knee arthroplasty. J Bone Jt Surg. (2020) 102:101-9. doi: 10.2106/JBJS.19.00695

20. Bini S, Mahajan J. Clinical outcomes of remote asynchronous telerehabilitation are equivalent to traditional therapy following total knee arthroplasty: a randomized control study. J Telemed Telecare. (2017) 23:239-47. doi: 10.1177/1357633X16634518

21. Hinman RS, Nelligan RK, Bennell KL, Delany C. "Sounds a bit crazy, but it was almost more personal:" a qualitative study of patient and clinician experiences of physical therapist-prescribed exercise for knee osteoarthritis via skype. Arthritis Care Res (Hoboken). (2017) 69:183444. doi: $10.1002 /$ acr.23218

22. Azma K, RezaSoltani Z, Rezaeimoghaddam F, Dadarkhah A, Mohsenolhosseini S. Efficacy of tele-rehabilitation compared with office-based physical therapy in patients with knee osteoarthritis: a randomized clinical trial. J Telemed Telecare. (2018) 24:560-5. doi: 10.1177/1357633X17723368

23. Chang C-F, Lin K-C, Chen W-M, Jane S-W, Yeh S-H, Wang T-J. Effects of a home-based resistance training program on recovery from total hip replacement surgery: feasibility and pilot testing. J Nurs Res. (2017) 25:2130. doi: $10.1097 /$ inr. 0000000000000128

24. Eichler S, Rabe S, Salzwedel A, Müller S, Stoll J, Tilgner N, et al. Effectiveness of an interactive telerehabilitation system with home-based exercise training in patients after total hip or knee replacement: study protocol for a multicenter, superiority, no-blinded randomized controlled trial. Trials. (2017) 18:438. doi: 10.1186/s13063-017-2173-3

25. Eichler S, Salzwedel A, Rabe S, Mueller S, Mayer F, Wochatz M, et al. The effectiveness of telerehabilitation as a supplement to rehabilitation in patients after total knee or hip replacement: randomized controlled trial. JMIR Rehabil Assist Technol. (2019) 6:e14236. doi: 10.2196/14236

26. Tsvyakh AI, Hospodarskyy AJ. Telerehabilitation of patients with injuries of the lower extremities. Telemed e-Health. (2017) 23:1011-5. doi: 10.1089/tmj.2016.0267

27. Pastora-Bernal J-M, Martín-Valero R, Barón-López FJ, García-Gómez O. Effectiveness of telerehabilitation programme following surgery in shoulder impingement syndrome (SIS): study protocol for a randomized controlled non-inferiority trial. Trials. (2017) 18:82. doi: 10.1186/s13063-017-1822-x

28. Pastora-Bernal JM, Martín-Valero R, Barón-López FJ, Moyano NG, Estebanez-Pérez M-J. Telerehabilitation after arthroscopic subacromial decompression is effective and not inferior to standard practice: preliminary results. J Telemed Telecare. (2018) 24:428-33. doi: 10.1177/1357633X17706583

29. Choi Y, Nam J, Yang D, Jung W, Lee H-R, Kim SH. Effect of smartphone application-supported self-rehabilitation for frozen shoulder: a prospective randomized control study. Clin Rehabil. (2019) 33:65360. doi: $10.1177 / 0269215518818866$

30. Santello G, Rossi DM, Martins J, Libardoni T de C, de Oliveira AS. Effects on shoulder pain and disability of teaching patients with shoulder pain a homebased exercise program: a randomized controlled trial. Clin Rehabil. (2020) 34:1245-55. doi: 10.1177/0269215520930790

31. Malliaras P, Cridland K, Hopmans R, Ashton S, Littlewood C, Page R, et al. Internet and telerehabilitation-delivered management of rotator cuffrelated shoulder pain (INTEL Trial): randomized controlled pilot and feasibility trial. JMIR mHealth uHealth. (2020) 8:e24311. doi: 10.2196/ 24311

32. Eriksson L, Lindström B, Gard G, Lysholm J. Physiotherapy at a distance: a controlled study of rehabilitation at home after a shoulder joint operation. J Telemed Telecare. (2009) 15:215-20. doi: 10.1258/jtt.2009.0 81003

33. Eriksson L, Lindström B, Ekenberg L. Patients' experiences of telerehabilitation at home after shoulder joint replacement. $J$ Telemed Telecare. (2011) 17:25-30. doi: 10.1258/jtt.2010.100317
34. Tousignant M, Giguère A-M, Morin M, Pelletier J, Sheehy A, Cabana F. Inhome telerehabilitation for proximal humerus fractures: a pilot study. Int $J$ Telerehabil. (2015) 6:31-8. doi: 10.5195/ijt.2014.6158

35. Steiner B, Elgert L, Saalfeld B, Schwartze J, Borrmann HP, Kobelt-Pönicke A, et al. Health-enabling technologies for telerehabilitation of the shoulder: a feasibility and user acceptance study. Methods Inf Med. (2020) 59:e90e9. doi: 10.1055/s-0040-1713685

36. Giuliano C, Parmenter BJ, Baker MK, Mitchell BL, Williams AD, Lyndon $\mathrm{K}$, et al. Cardiac rehabilitation for patients with coronary artery disease: a practical guide to enhance patient outcomes through continuity of care. Clin Med Insights Cardiol. (2017) 11:117954681771002. doi: 10.1177/1179546817710028

37. Williams MA. EXERCISE TESTING IN CARDIAC REHABILITATION: exercise prescription and beyond. Cardiol Clin. (2001) 19:41531. doi: 10.1016/S0733-8651(05)70226-5

38. Anderson L, Sharp GA, Norton RJ, Dalal H, Dean SG, Jolly K, et al. Homebased versus centre-based cardiac rehabilitation. Cochrane Database Syst Rev. (2017) 6:CD007130: doi: 10.1002/14651858.CD007130.pub4

39. Maddison R, Rawstorn JC, Rolleston A, Whittaker R, Stewart R, Benatar $\mathrm{J}$, et al. The remote exercise monitoring trial for exercise-based cardiac rehabilitation (REMOTE-CR): a randomised controlled trial protocol. $B M C$ Public Health. (2014) 14:1236. doi: 10.1186/1471-2458-14-1236

40. Maddison R, Rawstorn JC, Stewart RAH, Benatar J, Whittaker R, Rolleston A, et al. Effects and costs of real-time cardiac telerehabilitation: randomised controlled non-inferiority trial. Heart. (2019) 105:1229. doi: 10.1136/heartjnl-2018-313189

41. Rawstorn JC, Gant N, Rolleston A, Whittaker R, Stewart R, Benatar $\mathrm{J}$, et al. End users want alternative intervention delivery models: usability and acceptability of the REMOTE-CR exercise-based cardiac telerehabilitation program. Arch Phys Med Rehabil. (2018) 99:23737. doi: 10.1016/j.apmr.2018.06.027

42. Kraal JJ, Peek N, van den Akker-Van Marle ME, Kemps HMC. Effects and costs of home-based training with telemonitoring guidance in low to moderate risk patients entering cardiac rehabilitation: the FIT@Home study. BMC Cardiovasc Disord. (2013) 13:82. doi: 10.1186/1471-2261-13-82

43. Kraal JJ, Van den Akker-Van Marle ME, Abu-Hanna A, Stut W, Peek N, Kemps HMC. Clinical and cost-effectiveness of home-based cardiac rehabilitation compared to conventional, centre-based cardiac rehabilitation: results of the FIT@Home study. Eur J Prev Cardiol. (2017) 24:126073. doi: $10.1177 / 2047487317710803$

44. Hwang R, Bruning J, Morris NR, Mandrusiak A, Russell T. Home-based telerehabilitation is not inferior to a centre-based program in patients with chronic heart failure: a randomised trial. J Physiother. (2017) 63:1017. doi: 10.1016/j.jphys.2017.02.017

45. Hwang R, Morris NR, Mandrusiak A, Bruning J, Peters R, Korczyk D, et al. Cost-utility analysis of home-based telerehabilitation compared with centrebased rehabilitation in patients with heart failure. Hear Lung Circ. (2019) 28:1795-803. doi: 10.1016/j.hlc.2018.11.010

46. Bravo-Escobar R, González-Represas A, Gómez-González AM, MontielTrujillo A, Aguilar-Jimenez R, Carrasco-Ruíz R, et al. Effectiveness and safety of a home-based cardiac rehabilitation programme of mixed surveillance in patients with ischemic heart disease at moderate cardiovascular risk: a randomised, controlled clinical trial. BMC Cardiovasc Disord. (2017) 17:66. doi: 10.1186/s12872-017-0499-0

47. Fang J, Huang B, Xu D, Li J, Au WW. Innovative application of a homebased and remote sensing cardiac rehabilitation protocol in Chinese patients after percutaneous coronary intervention. Telemed e-Health. (2019) 25:28893. doi: $10.1089 / \mathrm{tmj} .2018 .0064$

48. Maddison R, Whittaker R, Stewart R, Kerr A, Jiang Y, Kira G, et al. HEART: heart exercise and remote technologies: a randomized controlled trial study protocol. BMC Cardiovasc Disord. (2011) 11:26. doi: 10.1186/1471-2261-11-26

49. Pfaeffli Dale L, Whittaker R, Dixon R, Stewart R, Jiang Y, Carter $\mathrm{K}$, et al. Acceptability of a mobile health exercise-based cardiac rehabilitation intervention. J Cardiopulm Rehabil Prev. (2015) 35:312-9. doi: 10.1097/HCR.0000000000000125

50. Maddison R, Pfaeffli L, Stewart R, Kerr A, Jiang Y, Rawstorn J, et al. The HEART mobile phone trial: the partial mediating effects of self-efficacy 
on physical activity among cardiac patients. Front Public Heal. (2014) 2:56. doi: 10.3389/fpubh.2014.00056

51. Maddison R, Pfaeffli L, Whittaker R, Stewart R, Kerr A, Jiang Y, et al. A mobile phone intervention increases physical activity in people with cardiovascular disease: results from the HEART randomized controlled trial. Eur J Prev Cardiol. (2015) 22:701-9. doi: 10.1177/2047487314535076

52. Frederix I, Hansen D, Coninx K, Vandervoort P, Van Craenenbroeck EM, Vrints C, et al. Telerehab III: a multi-center randomized, controlled trial investigating the long-term effectiveness of a comprehensive cardiac telerehabilitation program - Rationale and study design. BMC Cardiovasc Disord. (2015) 15:29. doi: 10.1186/s12872-015-0021-5

53. Frederix I, Solmi F, Piepoli MF, Dendale P. Cardiac telerehabilitation: a novel cost-efficient care delivery strategy that can induce long-term health benefits. Eur J Prev Cardiol. (2017) 24:1708-17. doi: 10.1177/204748 7317732274

54. Gloeckl R, Marinov B, Pitta F. Practical recommendations for exercise training in patients with COPD. Eur Respir Rev. (2013) 22:17886. doi: 10.1183/09059180.00000513

55. Borghi-Silva A, Arena R, Castello V, Simões RP, Martins LEB, Catai AM, et al. Aerobic exercise training improves autonomic nervous control in patients with COPD. Respir Med. (2009) 103:1503-10. doi: 10.1016/j.rmed.2009.04.015

56. Brønstad E, Tjonna AE, Rognmo $\varnothing$, Dalen H, Heggli AM, Wisloff U, et al. Aerobic exercise training improves right- and left ventricular systolic function in patients with COPD. COPD J Chronic Obstr Pulm Dis. (2013) 10:300-6. doi: 10.3109/15412555.2012.745843

57. Liao W-h, Chen J-w, Chen X, Lin L, Yan H-y, Zhou Y-q, et al. Impact of resistance training in subjects with COPD: a systematic review and meta-analysis. Respir Care. (2015) 60:1130-45. doi: 10.4187/respcare.03598

58. Vogiatzis I, Nanas S, Roussos C. Interval training as an alternative modality to continuous exercise in patients with COPD. Eur Respir J. (2002) 20:129. doi: 10.1183/09031936.02.01152001

59. Gosselink R, De Vos J, van den Heuvel SP, Segers J, Decramer M, Kwakkel G. Impact of inspiratory muscle training in patients with COPD: what is the evidence? Eur Respir J. (2011) 37:416-25. doi: 10.1183/09031936.00031810

60. Holland AE, Mahal A, Hill CJ, Lee AL, Burge AT, Moore R, et al. Benefits and costs of home-based pulmonary rehabilitation in chronic obstructive pulmonary disease - a multi-centre randomised controlled equivalence trial. BMC Pulm Med. (2013) 13:57. doi: 10.1186/1471-2466-13-57

61. Holland AE, Mahal A, Hill CJ, Lee AL, Burge AT, Cox NS, et al. Home-based rehabilitation for COPD using minimal resources: a randomised, controlled equivalence trial. Thorax. (2017) 72:57-65. doi: 10.1136/thoraxjnl-2016-208514

62. Hansen $H$, Bieler $T$, Beyer $N$, Godtfredsen $N$, Kallemose $T$, Frølich A. COPD online-rehabilitation versus conventional COPD rehabilitation - rationale and design for a multicenter randomized controlled trial study protocol (CORe trial). BMC Pulm Med. (2017) 17:140. doi: 10.1186/s12890-017-0488-1

63. Hansen H, Bieler T, Beyer N, Kallemose T, Wilcke JT, Østergaard LM, Frost Andeassen $\mathrm{H}$, et al. Supervised pulmonary tele-rehabilitation versus pulmonary rehabilitation in severe COPD: a randomised multicentre trial. Thorax. (2020) 75:413-21. doi: 10.1136/thoraxjnl-2019-2 14246

64. Godtfredsen N, Frølich A, Bieler T, Beyer N, Kallemose T, Wilcke $\mathrm{T}$, et al. 12-months follow-up of pulmonary tele-rehabilitation versus standard pulmonary rehabilitation: a multicentre randomised clinical trial in patients with severe COPD. Respir Med. (2020) 172:106129. doi: 10.1016/j.rmed.2020.106129

65. Chaplin E, Hewitt S, Apps L, Bankart J, Pulikottil-Jacob R, Boyce S, et al. Interactive web-based pulmonary rehabilitation programme: a randomised controlled feasibility trial. BMJ Open. (2017) 7:e013682. doi: 10.1136/bmjopen-2016-013682

66. Bourne S, DeVos R, North M, Chauhan A, Green B, Brown T, et al. Online versus face-to-face pulmonary rehabilitation for patients with chronic obstructive pulmonary disease: randomised controlled trial. BMJ Open. (2017) 7:e014580. doi: 10.1136/bmjopen-2016-014580

67. Cameron-Tucker H, Wood-Baker R, Joseph L, Walters J, Schuz N, Walters EH. A randomized controlled trial of telephone-mentoring with home-based walking preceding rehabilitation in COPD. Int J Chron Obstruct Pulmon Dis. (2016) 11:1991-2000. doi: 10.2147/COPD.S109820

68. Vasilopoulou M, Papaioannou AI, Kaltsakas G, Louvaris Z, Chynkiamis N, Spetsioti S, et al. Home-based maintenance telerehabilitation reduces the risk for acute exacerbations of COPD, hospitalisations and emergency department visits. Eur Respir J. (2017) 49:1602129. doi: 10.1183/13993003.02129-2016

69. Tabak M, Vollenbroek-Hutten MMR, van der Valk PD, van der Palen J, Hermens HJ. A telerehabilitation intervention for patients with Chronic Obstructive Pulmonary Disease: a randomized controlled pilot trial. Clin Rehabil. (2014) 28:582-91. doi: 10.1177/0269215513512495

70. van der Molen T, Willemse BWM, Schokker S, ten Hacken NHT, Postma DS, Juniper EF. Development, validity and responsiveness of the Clinical COPD Questionnaire. Health Qual Life Outcomes. (2003) 1:13. doi: 10.1186/1477-7525-1-13

71. Tsai LLY, McNamara RJ, Moddel C, Alison JA, McKenzie DK, McKeough ZJ. Home-based telerehabilitation via real-time videoconferencing improves endurance exercise capacity in patients with COPD: The randomized controlled TeleR Study. Respirology. (2017) 22:699-707. doi: 10.1111/resp.12966

72. Holland AE, Hill CJ, Rochford P, Fiore J, Berlowitz DJ, Mcdonald CF. Telerehabilitation for people with chronic obstructive pulmonary disease: feasibility of a simple, real time model of supervised exercise training. $J$ Telemed Telecare. (2013) 19:222-6. doi: 10.1177/1357633x13487100

73. Zanaboni P, Hoaas H, Aarøen Lien L, Hjalmarsen A, Wootton R. Long-term exercise maintenance in COPD via telerehabilitation: a two-year pilot study. J Telemed Telecare. (2017) 23:74-82. doi: 10.1177/1357633X15625545

74. Billinger SA, Arena R, Bernhardt J, Eng JJ, Franklin BA, Johnson CM, et al. Physical activity and exercise recommendations for stroke survivors. Stroke. (2014) 45:2532-53. doi: 10.1161/STR.0000000000000022

75. Pang MYC, Eng JJ, Dawson AS, Gylfadóttir S. The use of aerobic exercise training in improving aerobic capacity in individuals with stroke: a metaanalysis. Clin Rehabil. (2006) 20:97-111. doi: 10.1191/0269215506cr926oa

76. Harris JE, Eng JJ. Strength training improves upper-limb function in individuals with stroke. Stroke. (2010) 41:13640. doi: 10.1161/STROKEAHA.109.567438

77. Saunders DH, Sanderson M, Hayes S, Johnson L, Kramer S, Carter DD, et al. Physical fitness training for stroke patients. Cochrane Database Syst Rev. (2020) 3:CD003316. doi: 10.1002/14651858.CD003316.pub7

78. Veerbeek JM, Koolstra M, Ket JCF, van Wegen EEH, Kwakkel G. Effects of augmented exercise therapy on outcome of gait and gait-related activities in the first 6 months after stroke. Stroke. (2011) 42:33115. doi: 10.1161/STROKEAHA.111.623819

79. Chen M-D, Rimmer JH. Effects of exercise on quality of life in stroke survivors. Stroke. (2011) 42:832-7. doi: 10.1161/STROKEAHA.110.607747

80. Zedlitz AMEE, Rietveld TCM, Geurts AC, Fasotti L. Cognitive and graded activity training can alleviate persistent fatigue after stroke. Stroke. (2012) 43:1046-51. doi: 10.1161/STROKEAHA.111.632117

81. Levy T, Laver K, Killington M, Lannin N, Crotty M. A systematic review of measures of adherence to physical exercise recommendations in people with stroke. Clin Rehabil. (2019) 33:535-45. doi: 10.1177/0269215518 811903

82. Chumbler NR, Quigley P, Li X, Morey M, Rose D, Sanford J, et al. Effects of telerehabilitation on physical function and disability for stroke patients. Stroke. (2012) 43:2168-74. doi: 10.1161/STROKEAHA.111.6 46943

83. Chumbler NR, Li X, Quigley P, Morey MC, Rose D, Griffiths P, et al. A randomized controlled trial on Stroke telerehabilitation: the effects on falls self-efficacy and satisfaction with care. J Telemed Telecare. (2015) 21:13943. doi: 10.1109/CTS.2014.6867601

84. Chen J, Jin W, Dong WS, Jin Y, Qiao FL, Zhou YF, et al. Effects of home-based telesupervising rehabilitation on physical function for stroke survivors with hemiplegia: a randomized controlled trial. Am J Phys Med Rehabil. (2017) 96:152-60. doi: 10.1097/PHM.0000000000000559

85. Chen J, Liu M, Sun D, Jin Y, Wang T, Ren C. Effectiveness and neural mechanisms of home-based telerehabilitation in patients with stroke based on fMRI and DTI. Medicine (Baltimore). (2018) 97:e9605. doi: 10.1097/MD.0000000000009605 
86. Chen J, Sun D, Zhang S, Shi Y, Qiao F, Zhou Y, et al. Effects of homebased telerehabilitation in patients with stroke. Neurology. (2020) 95:e2318e30. doi: 10.1212/WNL.0000000000010821

87. Kairy D, Veras M, Archambault P, Hernandez A, Higgins J, Levin MF, et al. Maximizing post-stroke upper limb rehabilitation using a novel telerehabilitation interactive virtual reality system in the patient's home: study protocol of a randomized clinical trial. Contemp Clin Trials. (2016) 47:49-53. doi: 10.1016/j.cct.2015.12.006

88. Norouzi-Gheidari N, Hernandez A, Archambault PS, Higgins J, Poissant L, Kairy D. Feasibility, safety and efficacy of a virtual reality exergame system to supplement upper extremity rehabilitation post-stroke: a pilot randomized clinical trial and proof of principle. Int J Environ Res Public Health. (2019) 17:113. doi: 10.3390/ijerph17010113

89. Lloréns R, Noé E, Colomer C, Alcañiz M. Effectiveness, usability, and cost-benefit of a virtual reality-based telerehabilitation program for balance recovery after stroke: a randomized controlled trial. Arch Phys Med Rehabil. (2015) 96:418-425.e2. doi: 10.1016/j.apmr.2014.10.019

90. Linder SM, Rosenfeldt AB, Bay RC, Sahu K, Wolf SL, Alberts JL. Improving quality of life and depression after stroke through telerehabilitation. Am J Occup Ther. (2015) 69:6902290020p1-p10. doi: 10.5014/ajot.2015.014498

91. Sarfo FS, Adusei N, Ampofo M, Kpeme FK, Ovbiagele B. Pilot trial of a tele-rehab intervention to improve outcomes after stroke in Ghana: a feasibility and user satisfaction study. J Neurol Sci. (2018) 387:947. doi: 10.1016/j.jns.2018.01.039

92. Szturm T, Imran Z, Pooyania S, Kanitkar A, Mahana B. Evaluation of a game based tele rehabilitation platform for in-home therapy of hand-arm function post stroke: feasibility study. PMßR. (2021) 13:4554. doi: 10.1002/pmrj.12354

93. Touillet A, Guesdon H, Bosser G, Beis J-M, Paysant J. Assessment of compliance with prescribed activity by hemiplegic stroke patients after an exercise programme and physical activity education. Ann Phys Rehabil Med. (2010) 53:250-65. doi: 10.1016/j.rehab.2010.03.005

94. Miller KK, Porter RE, DeBaun-Sprague E, Van Puymbroeck M, Schmid AA. Exercise after stroke: patient adherence and beliefs after discharge from rehabilitation. Top Stroke Rehabil. (2017) 24:142-8. doi: 10.1080/10749357.2016.1200292

95. Latimer-Cheung AE, Pilutti LA, Hicks AL, Martin Ginis KA, Fenuta AM, MacKibbon KA, et al. Effects of exercise training on fitness, mobility, fatigue, and health-related quality of life among adults with multiple sclerosis: a systematic review to inform guideline development. Arch Phys Med Rehabil. (2013) 94:1800-28.e3. doi: 10.1016/j.apmr.2013.04.020

96. Edwards T, Pilutti LA. The effect of exercise training in adults with multiple sclerosis with severe mobility disability: a systematic review and future research directions. Mult Scler Relat Disord. (2017) 16:319. doi: 10.1016/j.msard.2017.06.003

97. Bjartmar C, Trapp BD. Axonal and neuronal degeneration in multiple sclerosis: mechanisms and functional consequences. Curr Opin Neurol. (2001) 14:271-8. doi: 10.1097/00019052-200106000-00003

98. Dalgas U, Stenager E. Progressive resistance therapy is not the best way to rehabilitate deficits due to multiple sclerosis: no. Mult Scler J. (2014) 20:141-2. doi: 10.1177/1352458513513060

99. Motl RW, Sandroff BM. Benefits of exercise training in multiple sclerosis. Curr Neurol Neurosci Rep. (2015) 15:62. doi: 10.1007/s11910-015-0 585-6

100. Pearson M, Dieberg G, Smart N. Exercise as a therapy for improvement of walking ability in adults with multiple sclerosis: a meta-analysis. Arch Phys Med Rehabil. (2015) 96:1339-48.e7. doi: 10.1016/j.apmr.201 5.02.011

101. Snook EM, Motl RW. Effect of exercise training on walking mobility in multiple sclerosis: a meta-analysis. Neurorehabil Neural Repair. (2009) 23:108-16. doi: 10.1177/1545968308320641

102. Paltamaa J, Sjögren T, Peurala S, Heinonen A. Effects of physiotherapy interventions on balance in multiple sclerosis: a systematic review and metaanalysis of randomized controlled trials. J Rehabil Med. (2012) 44:81123. doi: 10.2340/16501977-1047

103. Asano M, Finlayson ML. Meta-analysis of three different types of fatigue management interventions for people with multiple sclerosis: exercise, education, and medication. Mult Scler Int. (2014) 2014:112. doi: $10.1155 / 2014 / 798285$

104. Pilutti LA, Greenlee TA, Motl RW, Nickrent MS, Petruzzello SJ. Effects of exercise training on fatigue in multiple sclerosis. Psychosom Med. (2013) 75:575-80. doi: 10.1097/PSY.0b013e31829b4525

105. Paul L, Coulter EH, Miller L, McFadyen A, Dorfman J, Mattison PGG. Webbased physiotherapy for people moderately affected with Multiple Sclerosis; Quantitative and qualitative data from a randomized, controlled pilot study. Clin Rehabil. (2014) 28:924-35. doi: 10.1177/0269215514527995

106. Paul L, Renfrew L, Freeman J, Murray H, Weller B, Mattison P, et al. Web-based physiotherapy for people affected by multiple sclerosis: a single blind, randomized controlled feasibility study. Clin Rehabil. (2019) 33:47384. doi: 10.1177/0269215518817080

107. Tallner A, Streber R, Hentschke C, Morgott M, Geidl W, Mäurer M, et al. Internet-supported physical exercise training for persons with multiple sclerosis-a randomised, controlled study. Int J Mol Sci. (2016) 17:1667. doi: 10.3390/ijms17101667

108. Keytsman C, Van Noten P, Spaas J, Nieste I, Van Asch P, Eijnde BO. Periodized home-based training: a new strategy to improve high intensity exercise therapy adherence in mildly affected patients with Multiple Sclerosis. Mult Scler Relat Disord. (2019) 28:91-7. doi: 10.1016/j.msard.2018.12.018

109. Finkelstein J, Lapshin O, Castro H, Cha E, Provance PG. Home-based physical telerehabilitation in patients with multiple sclerosis: a pilot study. J Rehabil Res Dev. (2008) 45:1361-74. doi: 10.1682/JRRD.2008.01.0001

110. Fjeldstad-Pardo C, Thiessen A, Pardo G. Telerehabilitation in multiple sclerosis: results of a randomized feasibility and efficacy pilot study. Int $J$ Telerehabil. (2018) 10:55-64. doi: 10.5195/ijt.2018.6256

111. Dibble LE, Addison O, Papa E. The effects of exercise on balance in persons with Parkinson's disease: a systematic review across the disability spectrum. J Neurol Phys Ther. (2009) 33:14-26. doi: 10.1097/NPT.0b013e3181990fcc

112. Schoneburg B, Mancini M, Horak F, Nutt JG. Framework for understanding balance dysfunction in Parkinson's disease. Mov Disord. (2013) 28:147482. doi: $10.1002 / \mathrm{mds} .25613$

113. Myers PS, McNeely ME, Pickett KA, Duncan RP, Earhart GM. Effects of exercise on gait and motor imagery in people with Parkinson disease and freezing of gait. Parkinsonism Relat Disord. (2018) 53:8995. doi: 10.1016/j.parkreldis.2018.05.006

114. Giladi N, Horak FB, Hausdorff JM. Classification of gait disturbances: distinguishing between continuous and episodic changes. Mov Disord. (2013) 28:1469-73. doi: 10.1002/mds. 25672

115. van der Kolk NM, King LA. Effects of exercise on mobility in people with Parkinson's disease. Mov Disord. (2013) 28:1587-96. doi: 10.1002/mds. 25658

116. Feng Y-S, Yang S-D, Tan Z-X, Wang M-M, Xing Y, Dong F, et al. The benefits and mechanisms of exercise training for Parkinson's disease. Life Sci. (2020) 245:117345. doi: 10.1016/j.lfs.2020.117345

117. van der Kolk NM, de Vries NM, Penko AL, van der Vlugt M, Mulder AA, Post B, et al. A remotely supervised home-based aerobic exercise programme is feasible for patients with Parkinson's disease: results of a small randomised feasibility trial. J Neurol Neurosurg Psychiatry. (2018) 89:1003-5. doi: 10.1136/jnnp-2017-315728

118. van der Kolk NM, de Vries NM, Kessels RPC, Joosten H, Zwinderman AH, Post B, et al. Effectiveness of home-based and remotely supervised aerobic exercise in Parkinson's disease: a double-blind, randomised controlled trial. Lancet Neurol. (2019) 18:998-1008. doi: 10.1016/S1474-4422(19)30285-6

119. Gandolfi M, Geroin C, Dimitrova E, Boldrini P, Waldner A, Bonadiman S, et al. Virtual reality telerehabilitation for postural instability in parkinson's Disease: a multicenter, single-blind, randomized, controlled trial. Biomed Res Int. (2017) 2017:1-11. doi: 10.1155/2017/7962826

120. Lai B, Bond K, Kim Y, Barstow B, Jovanov E, Bickel CS. Exploring the uptake and implementation of tele-monitored home-exercise programmes in adults with Parkinson's disease: a mixed-methods pilot study. J Telemed Telecare. (2020) 26:53-63. doi: 10.1177/1357633X18794315

121. Seidler KJ, Duncan RP, McNeely ME, Hackney ME, Earhart GM. Feasibility and preliminary efficacy of a telerehabilitation approach to group adapted tango instruction for people with Parkinson disease. J Telemed Telecare. (2017) 23:740-6. doi: 10.1177/1357633X16668092 
122. $\mathrm{Fu} J$, Wang $\mathrm{H}$, Deng $\mathrm{L}$, Li J. Exercise training promotes functional recovery after spinal cord injury. Neural Plast. (2016) 2016:1-7. doi: 10.1155/2016/4039580

123. Jacobs PL, Nash MS. Modes, benefits, and risks of voluntary and electrically induced exercise in persons with spinal cord injury. J Spinal Cord Med. (2001) 24:10-8. doi: 10.1080/10790268.2001.11753549

124. Nas K. Rehabilitation of spinal cord injuries. World J Orthop. (2015) 6:8. doi: $10.5312 /$ wjo.v6.i1.8

125. Lai B, Rimmer J, Barstow B, Jovanov E, Bickel CS. Teleexercise for persons with spinal cord injury: a mixed-methods feasibility case series. JMIR Rehabil Assist Technol. (2016) 3:e8. doi: 10.2196/rehab.5524

126. Nightingale TE, Rouse PC, Walhin J-P, Thompson D, Bilzon JLJ. Homebased exercise enhances health-related quality of life in persons with spinal cord injury: a randomized controlled trial. Arch Phys Med Rehabil. (2018) 99:1998-2006.e1. doi: 10.1016/j.apmr.2018.05.008

127. Dolbow DR, Gorgey AS, Ketchum JM, Moore JR, Hackett LA, Gater DR. Exercise adherence during home-based functional electrical stimulation cycling by individuals with spinal cord injury. Am J Phys Med Rehabil. (2012) 91:922-30. doi: 10.1097/PHM.0b013e318269d89f

128. Dolbow D, Gorgey A, Ketchum J, Gater D. Home-based functional electrical stimulation cycling enhances quality of life in individuals with spinal cord injury. Top Spinal Cord Inj Rehabil. (2013) 19:3249. doi: 10.1310/sci1904-324

129. Prochazka A, Kowalczewski J. A fully automated, quantitative test of upper limb function. J Mot Behav. (2015) 47:1928. doi: 10.1080/00222895.2014.953442

130. Kowalczewski J, Chong SL, Galea M, Prochazka A. In-home telerehabilitation improves tetraplegic hand function. Neurorehabil Neural Repair. (2011) 25:412-22. doi: 10.1177/1545968310394869
131. Van Straaten MG, Cloud BA, Morrow MM, Ludewig PM, Zhao KD. Effectiveness of home exercise on pain, function, and strength of manual wheelchair users with spinal cord injury: a high-dose shoulder program with telerehabilitation. Arch Phys Med Rehabil. (2014) 95:18107.e2. doi: 10.1016/j.apmr.2014.05.004

132. Kehn M, Kroll T. Staying physically active after spinal cord injury: a qualitative exploration of barriers and facilitators to exercise participation. BMC Public Health. (2009) 9:168. doi: 10.1186/1471-245 8-9-168

Conflict of Interest: The authors declare that the research was conducted in the absence of any commercial or financial relationships that could be construed as a potential conflict of interest.

Publisher's Note: All claims expressed in this article are solely those of the authors and do not necessarily represent those of their affiliated organizations, or those of the publisher, the editors and the reviewers. Any product that may be evaluated in this article, or claim that may be made by its manufacturer, is not guaranteed or endorsed by the publisher.

Copyright (C) 2022 Amorese and Ryan. This is an open-access article distributed under the terms of the Creative Commons Attribution License (CC BY). The use, distribution or reproduction in other forums is permitted, provided the original author(s) and the copyright owner(s) are credited and that the original publication in this journal is cited, in accordance with accepted academic practice. No use, distribution or reproduction is permitted which does not comply with these terms. 